\title{
DEMAZURE FLAGS, $q$-FIBONACCI POLYNOMIALS AND HYPERGEOMETRIC SERIES
}

\author{
REKHA BISWAL, VYJAYANTHI CHARI, AND DENIZ KUS
}

\begin{abstract}
We study a family of finite-dimensional representations of the hyperspecial parabolic subalgebra of the twisted affine Lie algebra of type $\mathrm{A}_{2}^{(2)}$. We prove that these modules admit a decreasing filtration whose sections are isomorphic to stable Demazure modules in an integrable highest weight module of sufficiently large level. In particular, we show that any stable level $\mathrm{m}^{\prime}$ Demazure module admits a filtration by level $m$ Demazure modules for all $m \geq m^{\prime}$. We define the graded and weighted generating functions which encode the multiplicity of a given Demazure module and establish a recursive formulae. In the case when $m^{\prime}=1,2$ and $m=2,3$ we determine these generating functions completely and show that they define hypergeoemetric series and that they are related to the $q$-Fibonacci polynomials defined by Carlitz.
\end{abstract}

\section{INTRODUCTION}

The study of Demazure modules in highest weight representations of Kac-Moody algebras has been of interest for a long time. A character formula for these modules, analogous to the Weyl character formula, was given in $[8,13,17]$. Combinatorial versions of the character of such modules were given in [16]. In the case of affine Lie algebras there is extensive literature in the case of level one highest weight integrable modules; here the level is the integer by which the canonical central element of the affine Lie algebra acts on the highest weight module. The work of Sanderson [19] for $A_{n}^{(1)}$ and the work of Ion [10] more generally, shows that the character of a particular family of level one Demazure modules (which we shall refer to as stable Demazure modules) is given by a specialization of Macdonad polynomials (in the untwisted simply-laced case) and by the specialization of the Koornwinder polynomial for the twisted affine Lie algebras.

In [11], A. Joseph introduced the notion of a module admitting a Demazure flag. He proved in the case of the quantized enveloping algebra associated to a simply-laced affine Lie algebra that the tensor product of a one-dimensional Demazure module by an arbitrary Demazure module admits a filtration whose successive quotients are isomorphic to Demazure modules. It was shown in [18] that an analogous result could be deduced from [11] for stable Demazure modules in the simply-laced untwisted affine Lie algebras.

In this paper we turn our attention to such questions in the case of twisted affine Lie algebras; the most interesting situation being the Lie algebra of type $A_{2 n}^{(2)}$ and we consider the corresponding rank one situation. The stable Demazure modules that we shall be interested in are those which admit an action of the hyperspecial parabolic subalgebra (denoted $\mathfrak{C} \mathfrak{g}$ ) of the affine Lie algebra. Our first result constructs a large family (which includes the Demazure modules) of finite-dimensional modules for $\mathfrak{C} \mathfrak{g}$ which admit a Demazure flag. Analogous results for $\mathrm{A}_{1}^{(1)}$ were established in [6] using results from [7]. In the current situation we use results from [14]; however we have to work much harder to establish the analogs of the results of [6] for two reasons. We have to contend with the fact

V.C was partially supported by DMS 1303052.

D.K was partially funded under the Institutional Strategy of the University of Cologne within the German Excellence Initiative. 
that $\mathrm{A}_{2}^{(2)}$ is a much more complicated algebra and we also have to prove additional representation theoretic results which were not established in [14].

The primary goal of this paper is to study the relationship between the theory of Demazure flags and Koornwinder polynomials, Ramanujan mock-theta functions, Carlitz $q$-Fibonacci polynomials and more generally the theory of hypergeometric series. These connections arise as follows; the algebra $\mathfrak{C} \mathfrak{g}$ is integer graded and the Demazure modules admit a compatible grading. The corresponding generating series of graded multiplicities, along with certain weighted versions of these are what provide the link between the modules and number theory and combinatorics.

As a first example, the non-symmetric Koornwinder polynomial $E_{-n}\left(q^{2}, t\right)$ at $t=\infty$ coincides with the graded character of $D(1, n)$ (for details see [10]). Our results show that we can express the specialized Koornwinder polynomial as a $\mathbb{N}[q]$-linear combination of graded characters of level $m$ Demazure modules for any fixed $m \geq 1$. The generating series of the graded multiplicities of the trivial module in a level three Demazure flag has interesting specializations; one of which gives rise to a fifth order mock theta function of Ramanujan. Analogous connections were made in the untwisted case in [2].

In this paper we introduce the weighted generating series of the multiplicities. Namely we define the weighted multiplicity of a Demazure module $D$ occuring in a Demazure flag of a module $V$ by multiplying the graded multiplicity by a power of $q$ so that the resulting polynomial is either zero or has a non-zero constant term. We show that in the case of level two flags in level one Demazure modules the resulting weighted generating series is a specialization of the hypergeometric function ${ }_{1} F_{1}\left[\begin{array}{ll}a \\ b\end{array} ; q\right]$. In the case of level three flags in level two Demazure modules, the generating series are determined explicitly and is essentially given by the $q$-Fibonacci polynomials defined by Carlitz. We remind the reader that the original $q$-analogs of the Fibonacci polynomials were introduced by Schur [20] in his work on the Rogers-Ramanujan identities; the latter identities are well-known to be related to the representation theory of affine Lie algebras, but in a very different context [15]. Moreover, we give a closed form for the generating series for the numerical multiplicities $(q=1)$ and find that they involve the Chebyshev polynomials of the second kind. Again analogous results along these lines were first proved in [2].

The case of higher level Demazure flags is much more complicated; however our results on weighted multiplicities do suggest that the hypergeometric series again appear, but this is still conjectural. The results of [2], the current paper and the work in progress [3], clearly indicate a deep and unexpected connection between the theory of Demazure flags and its combinatorics and number theory.

The paper is organized as follows. In Section 2 we state the main results of the paper with the minimum possible notation. The representation theoretic results are established in Sections 3 and Section 4. The last sections are devoted to using the representation theory to calculate the graded and weighted multiplicities.

Acknowledgment: The third author thanks George Andrews and Volker Genz for many helpful discussions.

\section{Preliminaries}

1.1. We denote the set of complex numbers by $\mathbb{C}$ and, respectively, the set of integers, nonnegative integers, and positive integers by $\mathbb{Z}, \mathbb{Z}_{+}$, and $\mathbb{N}$. We set $\mathbf{N}=\left\{(r, s): r, s \in \frac{1}{2} \mathbb{N}, r+s \in \mathbb{N}\right\}$ and let $y_{+}=\max \{0, y\}$ for $y \in \mathbb{R}$. All vector spaces considered in this paper are $\mathbb{C}$-vector spaces. For a $\mathbb{Z}$-graded vector space $V=\bigoplus_{k \in \mathbb{Z}} V[k]$ we denote by $\tau_{p}^{*} V$ the graded vector space whose $k$-th graded piece is $V[k+p]$. Given a complex Lie algebra $\mathfrak{a}$, we let $\mathbf{U}(\mathfrak{a})$ be the corresponding universal enveloping algebra. 
1.2. We refer to [12] for the general theory of affine Lie algebras. The focus of this paper is the twisted affine Lie algebra $\widehat{\mathfrak{g}}$ of type $\mathrm{A}_{2}^{(2)}$, which contains the simple Lie algebra $\mathfrak{g}=\mathfrak{s l}_{2}$ as a subalgebra. Recall that $\mathfrak{s l}_{2}$ is the complex simple Lie algebra of two by two matrices of trace zero and that $\left\{x_{0}, y_{0}, h_{0}\right\}$ is the standard basis with $\left[h_{0}, x_{0}\right]=2 x_{0},\left[h_{0}, y_{0}\right]=-2 y_{0}$ and $\left[x_{0}, y_{0}\right]=h_{0}$. The element $h_{0}$ generates a Cartan subalgebra $\mathfrak{h}$ of $\mathfrak{g}$ and let $R=\{ \pm \alpha\}$ be the set of roots with respect to $\mathfrak{h}$. We fix $\widehat{\mathfrak{h}}$ a Cartan subalgebra of $\widehat{\mathfrak{g}}$ containing $\mathfrak{h}$ and let $\widehat{R}$ the set of roots of $\widehat{\mathfrak{g}}$ with respect to $\widehat{\mathfrak{h}}$. The corresponding sets of positive and negative roots are denoted as usual by $\widehat{R}^{ \pm}$and $R^{ \pm}$respectively. If $\delta$ denotes the unique non-divisible positive imaginary root in $\widehat{R}$, then we have $\widehat{R}=\widehat{R}^{+} \cup \widehat{R}^{-}$, where $\widehat{R}^{-}=-\widehat{R}^{+}, \widehat{R}^{+}=\widehat{R}_{\mathrm{re}}^{+} \cup \widehat{R}_{\mathrm{im}}^{+}, \widehat{R}_{\mathrm{im}}^{+}=\mathbb{N} \delta$, and

$$
\widehat{R}_{\mathrm{re}}^{+}=R^{+} \cup(R+2 \mathbb{N} \delta) \cup \frac{1}{2}\left(R+\left(2 \mathbb{Z}_{+}+1\right) \delta\right) .
$$

We also consider the set

$$
\widehat{R}_{\mathrm{re}}( \pm)=R^{ \pm} \cup\left(R^{ \pm}+2 \mathbb{N} \delta\right) \cup \frac{1}{2}\left(R^{ \pm}+\left(2 \mathbb{Z}_{+}+1\right) \delta\right) .
$$

Given $\beta \in \widehat{R}$ let $\widehat{\mathfrak{g}}_{\beta} \subset \widehat{\mathfrak{g}}$ be the corresponding root space; note that $x_{0}$ (resp. $y_{0}$ ) is a generator of the root space $\widehat{\mathfrak{g}}_{\alpha}\left(\right.$ resp. $\left.\widehat{\mathfrak{g}}_{-\alpha}\right)$. For any real root $\beta$ we fix a generator $x_{\beta}$ of $\widehat{\mathfrak{g}}_{\beta}$ and abbreviate

$$
x_{\alpha+2 r \delta}:=x_{2 r}, \quad x_{\frac{\alpha}{2}+\left(r+\frac{1}{2}\right) \delta}:=x_{r+\frac{1}{2}}, \quad x_{-\alpha+2 r \delta}:=y_{2 r}, \quad x_{-\frac{\alpha}{2}+\left(r+\frac{1}{2}\right) \delta}:=y_{r+\frac{1}{2}} .
$$

1.3. We define several subalgebras of $\widehat{\mathfrak{g}}$ that will be needed in the rest of the paper. Let $\widehat{\mathfrak{b}}$ be the Borel subalgebra corresponding to $\widehat{R}^{+}$, and let $\widehat{\mathfrak{n}}^{+}$be its nilpotent radical,

$$
\widehat{\mathfrak{b}}=\widehat{\mathfrak{h}} \oplus \widehat{\mathfrak{n}}^{+}, \quad \widehat{\mathfrak{n}}^{ \pm}=\bigoplus_{\beta \in \widehat{R}^{+}} \widehat{\mathfrak{g}}_{ \pm \beta} .
$$

The subalgebras $\mathfrak{b}$ and $\mathfrak{n}^{ \pm}$of $\mathfrak{g}$ are defined similarly. The twisted current algebra $\mathfrak{C} \mathfrak{g}$ is defined as

$$
\mathfrak{C} \mathfrak{g}=\mathfrak{h} \oplus \widehat{\mathfrak{n}}^{+} \oplus \mathfrak{n}^{-}
$$

and admits a triangular decomposition

$$
\mathfrak{C} \mathfrak{g}=\mathfrak{C n}^{+} \oplus \mathfrak{C h} \oplus \mathfrak{C n}^{-}
$$

where

$$
\mathfrak{C h}=\mathfrak{C h}_{+} \oplus \mathfrak{h}, \quad \mathfrak{C h}_{+}=\bigoplus_{k>0} \widehat{\mathfrak{g}}_{k \delta}, \quad \mathfrak{C n}^{ \pm}=\bigoplus_{\beta \in \widehat{R}_{\mathrm{re}}( \pm)} \widehat{\mathfrak{g}}_{ \pm \beta}
$$

Following [5] we call $\mathfrak{C} \mathfrak{g}$ the hyperspecial twisted current algebra, which is different from the notion of twisted current algebras of type $\mathrm{A}_{2}^{(2)}$ that exists in the literature. The differences are clarified in [5, Remark 2.5]. To simplify notation we set

$$
\mathbf{U}(\mathfrak{C} \mathfrak{g}):=\mathbf{U}, \quad \mathbf{U}\left(\mathfrak{C n}^{ \pm}\right):=\mathbf{U}^{ \pm}
$$

1.4. The scaling operator $d \in \widehat{\mathfrak{h}}$ defines a $\mathbb{Z}_{+}$-graded Lie algebra structure on $\mathfrak{C} \mathfrak{g}$ : for $\beta \in \widehat{R}$ we say that $\widehat{\mathfrak{g}}_{\beta}$ has grade $k$ if $\beta(d)=k$. Since $\delta(d)=2$ the eigenvalues of $d$ are all integers and if $\mathfrak{g}_{\beta} \subset \mathfrak{C} \mathfrak{g}$, then the eigenvalues are non-negative integers. With respect to this grading, the zero homogeneous component of the twisted current algebra is $\mathfrak{C} \mathfrak{g}[0]=\mathfrak{g}$. A finite-dimensional $\mathbb{Z}_{+}$-graded $\mathfrak{C} \mathfrak{g}$-module is a $\mathbb{Z}$-graded vector space admitting a compatible graded action of $\mathfrak{C} \mathfrak{g}$ :

$$
V=\bigoplus_{k \in \mathbb{Z}} V[k], \quad \mathfrak{C} \mathfrak{g}[r] V[k] \subset V[k+r] .
$$


Note that each graded component $V[k]$ is a $\mathfrak{g}$-module and we define the graded character as

$$
\operatorname{ch}_{\mathrm{gr}} V=\sum_{k \in \mathbb{Z}} \operatorname{ch}_{\mathfrak{g}} V[k] q^{k} .
$$

\section{The MAIN RESUlts}

We summarize the main results of the paper. We keep the notation to a minimum and refer the reader to the later sections for precise definitions.

2.1. Given $m \in \mathbb{N}$ and $n \in \mathbb{Z}_{+}$with $n=n_{1} m+n_{0}$, where $n_{0}, n_{1} \in \mathbb{Z}, 0<n_{0} \leq m$, let $D(m, n)$ be the graded $\mathfrak{C} \mathfrak{g}$-module generated by an element $v_{n}$ with defining relations:

$$
\begin{aligned}
& \left(\mathfrak{C n}^{+} \oplus \mathfrak{C h}_{+}\right) v_{n}=0, \quad h_{0} v_{n}=n v_{n}, \quad y_{0}^{n+1} v_{n}=0 \\
& y_{2 n_{1}+2} v_{n}=0, \quad y_{n_{1}+\frac{3}{2}} v_{n}=0, \quad \text { if } m>1 \\
& y_{2 n_{1}}^{n_{0}+1} v_{n}=0, \quad y_{n_{1}+\frac{1}{2}}^{\left(2 n_{0}-m\right)_{+}+1} v_{n}=0, \quad \text { if } n_{0}<m .
\end{aligned}
$$

It was proved in [14] that $D(m, n)$ is a finite-dimensional indecomposable $\mathfrak{C} \mathfrak{g}$-module and is isomorphic to a Demazure module occurring in a highest weight integrable irreducible representation of $\widehat{\mathfrak{g}}$. We call $m$ the level of the Demazure module.

2.2. We define the notion of a Demazure flag as follows. Let $V$ be a graded finite-dimensional $\mathfrak{C} \mathfrak{g}$-module $V$; we say that $V$ admits a a level $m$-Demazure flag if there exists a decreasing sequence of graded $\mathfrak{C} \mathfrak{g}$-submodules of $V$

$$
\mathcal{F}(V)=\left(0 \subset V_{0} \subset V_{1} \subset \cdots \subset V_{k}=V\right)
$$

such that the successive quotients of the flag are isomorphic to $\tau_{p}^{*} D(m, n)$ for some $n, p \geq 0$.

Let $\left[V: \tau_{p}^{*} D(m, n)\right]$ be the number of times $\tau_{p}^{*} D(m, n)$ occurs as a section of this flag. It is not hard to see that this number is independent of the choice of the flag. For an indeterminate $q$, we define a polynomial in $\mathbb{N}[q]$ by

$$
[V: D(m, n)]_{q}=\sum_{p \geq 0}\left[V: \tau_{p}^{*} D(m, n)\right] q^{p}
$$

We also set

$$
[V: D(m, n)]_{q}:=0 \text {, if } n<0 \text {. }
$$

We call $[V: D(m, n)]_{q}$ the graded multiplicity of $D(m, n)$ in $V$. If $[V: D(m, n)]_{q}$ is non-zero we define the weighted multiplicity of $D(m, n)$ in $V$ to be the unique polynomial $[V: D(m, n)]_{q}^{w}$ in $\mathbb{N}[q]$ with non-zero constant term satisfying

$$
q^{r}[V: D(m, n)]_{q}^{w}=[V: D(m, n)]_{q} \text { for some } r \in \mathbb{Z}_{+} .
$$

Otherwise we set $[V: D(m, n)]_{q}^{w}=[V: D(m, n)]_{q}=0$.

2.3. The first result of our paper is the following:

Theorem 1. For all integers $m \geq m^{\prime}>0$ and $s \geq 0$ the module $D\left(m^{\prime}, s\right)$ admits a Demazure flag of level $m$. Moreover,

$$
\left[D\left(m^{\prime}, s\right): D(m, n)\right]_{q}=\delta_{n, s}, \text { if } n \geq s, \quad[D(m, s): D(m, n)]_{q}=\delta_{n, s}
$$

and for $m \geq \ell \geq m^{\prime}>0$ we have

$$
\left[D\left(m^{\prime}, s\right): D(m, n)\right]_{q}=\sum_{p \geq 0}\left[D\left(m^{\prime}, s\right): D(\ell, p)\right]_{q}[D(\ell, p): D(m, n)]_{q} .
$$


Remark. In the case of quantized enveloping algebras associated with simply-laced Kac-Moody Lie algebras the existence of such a flag was proved in [11] using the theory of canonical bases. Later, it was shown in [18] that taking the classical limit, the result remains true for the corresponding affine Lie algebras.

An alternate constructive proof was given in [6] in the case of $A_{1}^{(1)}$; this proof enables one to compute multiplicities in the Demazure flag. We follow this approach in the current paper; however there are many non-trivial representation theoretic results that have to first be established for $\mathfrak{C} \mathfrak{g}$. In particular, we shall prove in Section 4 that a more general family of modules admit a Demazure flag.

2.4. Our next results deal with understanding the graded and weighted multiplicities of a level $m$ Demazure flag in $D\left(m^{\prime}, n\right)$ in the case when $\left.\left(m^{\prime}, m\right) \in\{1,2),(2,3)\right\}$. Given $n \in \mathbb{Z}_{+}$and $m \in \mathbb{Z}$, the $q$-binomial coefficient is defined by

$$
\left[\begin{array}{c}
n \\
m
\end{array}\right]_{q}=\frac{(q ; q)_{n}}{(q ; q)_{n-m}(q ; q)_{m}}, \quad n \geq m>0, \quad\left[\begin{array}{l}
n \\
0
\end{array}\right]_{q}=1, \quad\left[\begin{array}{c}
n \\
m
\end{array}\right]_{q}=0, \quad m<0 \text { or } m>n
$$

where the $q$-Pochammer symbol $(a ; q)_{n}$ is defined as

$$
(a ; q)_{n}=\prod_{i=1}^{n}\left(1-a q^{i-1}\right), n>0,(a ; q)_{0}=1 .
$$

Recall the follwing well-known $q$-binomial identities:

$$
\left[\begin{array}{l}
n \\
m
\end{array}\right]_{q}=\left[\begin{array}{c}
n-1 \\
m
\end{array}\right]_{q}+q^{n-m}\left[\begin{array}{c}
n-1 \\
m-1
\end{array}\right]_{q}, \quad\left[\begin{array}{l}
n \\
m
\end{array}\right]_{q}=q^{m}\left[\begin{array}{c}
n-1 \\
m
\end{array}\right]_{q}+\left[\begin{array}{c}
n-1 \\
m-1
\end{array}\right]_{q} .
$$

For $s \in \mathbb{Z}_{+}$let $\operatorname{res}_{2}(s) \in\{0,1\}$ be defined by requiring $s-\operatorname{res}_{2}(s)$ be even. The proof of the next proposition can be found in Section 5 .

Proposition. Let $s, p \in \mathbb{Z}_{+}$.

(i) We have,

$$
[D(1, s+p): D(2, s)]_{q}^{w}=\left[\begin{array}{c}
\left\lfloor\frac{s}{2}\right\rfloor+p \\
p
\end{array}\right]_{q^{2}},
$$

and

$$
D(1, s+p): D(2, s)]_{q}=q^{p\left(s+p+\operatorname{res}_{2}(s)\right)}[D(1, s+p): D(2, s)]_{q}^{w} .
$$

(ii) For $0 \leq r \leq 5$, let

$$
r^{\prime}=\delta_{r, 1}+\delta_{r, 4}, \quad \bar{r}=\left(\delta_{r, 1}+\delta_{r, 3}+\delta_{r, 5}\right) \operatorname{res}_{2}(p)-\delta_{r, 1}, \quad \tilde{r}=\left\lfloor\frac{r}{3}\right\rfloor .
$$

Setting $n=6 s+r$, we have

$$
[D(2, n+p): D(3, n)]_{q}^{w}=\sum_{j=0}^{\left\lfloor\frac{p}{2}\right\rfloor} q^{2 j\left(j+r^{\prime}+\operatorname{res}_{2}(p)\right)}\left[\begin{array}{c}
2 s+\tilde{r}+\left\lfloor\frac{p}{2}\right\rfloor-j \\
2 s+\tilde{r}
\end{array}\right]_{q^{2}}\left[\begin{array}{c}
s+j+\bar{r} \\
\left.2 j+\operatorname{res}_{2}(p)\right]_{q^{2}}
\end{array}\right.
$$

and

$$
[D(2, n+p): D(3, n)]_{q}=q^{p\left(4 s+r-\tilde{r}+\left\lceil\frac{p}{2}\right\rceil\right)+\operatorname{res}_{2}(p)\left(r^{\prime}+\tilde{r}-\left\lceil\frac{p}{2}\right\rceil\right)}[D(2, n+p): D(3, n)]_{q}^{w} .
$$

Remark. One outcome of our results is the following. It was proved in [10] that the specialization of the non-symmetric Koornwinder polynomial $E_{-n}\left(q^{2}, t\right)$ at $t=\infty$ coincides with the graded character of $D(1, n)$. Using Theorem 1 with $m^{\prime}=1$, we see that we can express $E_{-n}\left(q^{2}, t\right)$ as a $\mathbb{N}[q]$-linear combination of graded characters of level $m$ Demazure modules. In the case when $m^{\prime}=1$ and $m=2,3$ our analyses gives closed formulae for this decomposition of Koornwinder polynomials. 
2.5. Given $n \in \mathbb{Z}, m^{\prime}, m \in \mathbb{N}$ with $n \geq 0$ and $m \geq m^{\prime}$ we define generating series which encode the graded and weighted multiplicities of a level $m$ flag in a level $m^{\prime}$ Demazure module:

$A_{n}^{m^{\prime} \rightarrow m}(x, q):=\sum_{p \geq 0}\left[D\left(m^{\prime}, n+p\right): D(m, n)\right]_{q} x^{p}, \quad A_{n}^{m^{\prime} \rightarrow m, w}(x, q):=\sum_{p \geq 0}\left[D\left(m^{\prime}, n+p\right): D(m, n)\right]_{q}^{w} x^{p}$.

We shall relate these series to general basic hypergeometric series defined by

$$
{ }_{r} F_{s}\left[\begin{array}{lll}
a_{1} & \ldots & a_{r} \\
b_{1} & \ldots & b_{s}
\end{array} ; q z\right]=\sum_{n \geq 0} \frac{\left(a_{1} ; q\right)_{n}\left(a_{2} ; q\right)_{n} \cdots\left(a_{r} ; q\right)_{n}}{\left(b_{1} ; q\right)_{n}\left(b_{2} ; q\right)_{n} \cdots\left(b_{s} ; q\right)_{n}} \frac{z^{n}}{(q ; q)_{n}} .
$$

For more details and properties of hypergeometric series we refer the reader to [21].

2.5.1. Consider the case when $\left(m^{\prime}, m\right)=(1,2)$. Proposition 2.4(i) gives that

$$
[D(1,2 n+p): D(2,2 n)]_{q}^{w}=[D(1,2 n+1+p): D(2,2 n+1)]_{q}^{w},
$$

and hence we set

$$
\Phi_{n+1}^{1 \rightarrow 2}(x, q):=A_{2 n+1}^{1 \rightarrow 2, w}(x, q)=A_{2 n}^{1 \rightarrow 2, w}(x, q) .
$$

A further application of Proposition 2.4(i) gives

$$
\Phi_{n+1}^{1 \rightarrow 2}(x, q)=\sum_{p \geq 0}\left[\begin{array}{c}
n+p \\
n
\end{array}\right]_{q^{2}} x^{p}=\sum_{j \geq 0}\left[\begin{array}{l}
j \\
n
\end{array}\right]_{q^{2}} x^{j-n} .
$$

Using the identity

$$
\sum_{j \geq 0}\left[\begin{array}{l}
j \\
k
\end{array}\right]_{q} x^{j}=\frac{x^{k}}{(x: q)_{k+1}}
$$

we get

$$
\Phi_{n+1}^{1 \rightarrow 2}(x, q)=\frac{1}{\left(x ; q^{2}\right)_{n+1}} .
$$

It follows that if we set $\Phi_{0}^{1 \rightarrow 2}=1$, then

$$
\sum_{n \geq 0} \Phi_{n}^{1 \rightarrow 2}(x, q) z^{n}={ }_{1} F_{1}\left[\begin{array}{c}
q^{2} \\
x
\end{array} ; q^{2} z\right] .
$$

2.5.2. We now consider the case when $m=3$. In the case when $\left(m^{\prime}, m\right)=(1,3)$ we prove the following,

Proposition. We have

$$
A_{0}^{1 \rightarrow 3}(1, q)=\phi_{0}(q), \quad q A_{1}^{1 \rightarrow 3}(1, q)=\phi_{1}(q)
$$

where

$$
\phi_{0}(q)=\sum_{n \geq 0} q^{n^{2}}\left(-q ; q^{2}\right)_{n}, \quad \phi_{1}(q)=\sum_{n \geq 0} q^{(n+1)^{2}}\left(-q ; q^{2}\right)_{n},
$$

are the fifth order mock-theta functions of Ramanujan.

Proof. Using equation (2.7) we see that

$$
A_{0}^{1 \rightarrow 3}(x, q)=\sum_{p \geq 0}[D(1, p): D(3,0)]_{q} x^{p}=\sum_{p, s \geq 0}[D(1, p): D(2, s)]_{q}[D(2, s): D(3,0)]_{q} x^{p} .
$$

Equation (2.10) gives,

$$
[D(2,2 j+1): D(3,0)]_{q}=0, \quad[D(2,2 j): D(3,0)]=q^{2 j^{2}},
$$


and hence using Proposition 2.4(i) we get

$$
\begin{aligned}
A_{0}^{1 \rightarrow 3}(x, q) & =\sum_{p, j \geq 0} q^{2 j^{2}+(p-2 j) p}\left[\begin{array}{c}
p-j \\
p-2 j
\end{array}\right]_{q^{2}} x^{p} \\
& =\sum_{i, j \geq 0} q^{j^{2}+i^{2}}\left[\begin{array}{l}
i \\
j
\end{array}\right]_{q^{2}} x^{i+j} \\
& =\sum_{i \geq 0} q^{i^{2}} x^{i} \sum_{j \geq 0} q^{j^{2}-j}\left[\begin{array}{l}
i \\
j
\end{array}\right]_{q^{2}}(q x)^{j} \\
& =\sum_{i \geq 0} q^{i^{2}}\left(-q x ; q^{2}\right)_{i} x^{i} .
\end{aligned}
$$

Thus, $A_{0}^{1 \rightarrow 3}(1, q)=\phi_{0}(q)$. The proof in the other case is similar.

2.5.3. We now consider the case $\left(m^{\prime}, m\right)=(2,3)$. In this case the generating series $A_{n}^{2 \rightarrow 3, w}(x, q)$ is related to the $q$-Fibonacci polynomials defined by Carlitz in [4]:

$$
S_{n}(x, q)_{0}=x S_{n-1}(x, q)_{0}+q^{n-2} S_{n-2}(x, q)_{0}, \quad S_{0}(x, q)_{0}=0, \quad S_{1}(x, q)_{0}=1 .
$$

We remark that the specialization $S_{n}(1, q)_{0}$ was first considered by Schur [20] in his proof of the Rogers-Ramanujan identities; see also [1] for more details.

The solution to this recurrence is

$$
S_{n+1}(x, q)_{0}=\sum_{j \geq 0}\left[\begin{array}{c}
n-j \\
j
\end{array}\right]_{q} q^{j^{2}} x^{n-2 j} .
$$

The same recurrence relation but with different initial conditions,

$$
S_{n}(x, q)_{1}=x S_{n-1}(x, q)_{1}+q^{n-2} S_{n-2}(x, q)_{1}, \quad S_{-1}(x, q)_{1}=0, \quad S_{0}(x, q)_{1}=1,
$$

has the solution

$$
S_{n}(x, q)_{1}=\sum_{j \geq 0}\left[\begin{array}{c}
n-j \\
j
\end{array}\right]_{q} q^{j(j-1)} x^{n-2 j}
$$

Write

$$
A_{n}^{2 \rightarrow 3, w}(x, q)=A_{n}^{2 \rightarrow 3, w}(x, q)_{0}+A_{n}^{2 \rightarrow 3, w}(x, q)_{1},
$$

where

$$
A_{n}^{2 \rightarrow 3, w}(x, q)_{k}:=\sum_{p \geq 0}[D(2, n+2 p+k): D(3, n)]_{q}^{w} x^{2 p+k}, \quad k \in\{0,1\} .
$$

We prove,

Proposition. For $0 \leq r \leq 5$ we set

$$
s_{0}=s-\delta_{r, 1}, \quad s_{1}=s-1+\delta_{r, 3}+\delta_{r, 5} .
$$

Then,

$$
A_{6 s+r}^{2 \rightarrow 3, w}(x, q)_{k}=\frac{q^{-2 s_{k}^{2}-k}}{\left(x^{2} ; q^{2}\right)_{2 s+\left\lfloor\frac{r}{3}\right\rfloor}} S_{2 s_{k}+1}\left(y, q^{2}\right)_{k}, \quad k \in\{0,1\},
$$

where $y=q^{2 s_{k}+r^{\prime}} x$. 
Proof. Using the formulae in Proposition 2.4(ii) we get

$$
\begin{aligned}
A_{6 s+r}^{2 \rightarrow 3, w}(x, q)_{0} & =\sum_{p, j \geq 0} q^{2 j\left(j+r^{\prime}\right)}\left[\begin{array}{c}
2 s+\tilde{r}+p-j \\
2 s+\tilde{r}
\end{array}\right]_{q^{2}}\left[\begin{array}{c}
s+j+\bar{r} \\
2 j
\end{array}\right]_{q^{2}} x^{2 p} \\
& =\sum_{i \geq 0}\left[\begin{array}{c}
2 s+\tilde{r}+i \\
i
\end{array}\right]_{q^{2}} x^{2 i}\left(\sum_{j \geq 0} q^{2 j\left(j+r^{\prime}\right)}\left[\begin{array}{c}
s_{0}+j \\
2 j
\end{array}\right]_{q^{2}} x^{2 j}\right) \\
& =\frac{1}{\left(x^{2} ; q^{2}\right)_{2 s+\left\lfloor\frac{r}{3}\right\rfloor}}\left(\sum_{j \geq 0} q^{2 j\left(j+r^{\prime}\right)}\left[\begin{array}{c}
s_{0}+j \\
2 j
\end{array}\right]_{q^{2}} x^{2 j}\right) \\
& =\frac{q^{-2 s_{0}^{2}}}{\left(x^{2} ; q^{2}\right)_{2 s+\left\lfloor\frac{r}{3}\right\rfloor}} S_{2 s_{0}+1}\left(y, q^{2}\right)_{0} .
\end{aligned}
$$

The remaining case works similarly.

2.5.4. The generating series can also be viewed as limits of hypergeometric series and we thank George Andrews for helping us with this observation. In particular,

$$
\begin{aligned}
\left(x^{2} ; q^{2}\right)_{\left\lfloor\frac{n}{3}\right\rfloor+1} & A_{6 s+r}^{2 \rightarrow 3, w}(x, q)_{0} \\
& =\sum_{j \geq 0} q^{2 j\left(j+r^{\prime}\right)} \frac{(-1)^{j} q^{2 s_{0} j-j^{2}+j}\left(q^{2 s_{0}+2} ; q^{2}\right)_{j}\left(q^{-2 s_{0}} ; q^{2}\right)_{j}}{\left(q^{2} ; q^{2}\right)_{2 j}} x^{2 j} \\
& =\sum_{j \geq 0} q^{2 j\left(s_{0}+r^{\prime}\right)+j(j+1)}(-1)^{j} \frac{\left(q^{2 s_{0}+2} ; q^{2}\right)_{j}\left(q^{-2 s_{0}} ; q^{2}\right)_{j}}{\left(q^{2} ; q^{2}\right)_{j}\left(-q^{2} ; q^{2}\right)_{j}\left(q ; q^{2}\right)_{j}\left(-q ; q^{2}\right)_{j}} x^{2 j} \\
& =\lim _{t \rightarrow 0}{ }_{4} F_{3}\left[\begin{array}{c}
q / t t q^{2 s_{0}+2} q^{-2 s_{0}} ; q^{2} t x^{2} q^{2\left(s_{0}+r^{\prime}\right)+1} \\
-q^{2} q-q
\end{array}\right] .
\end{aligned}
$$

2.6. Our final collection of results discuss the generating functions for the numerical multiplicities, namely we set

$$
A_{n}^{m^{\prime} \rightarrow m}(x):=\sum_{p \geq 0}\left[D\left(m^{\prime}, n+p\right): D(m, n)\right]_{q=1} x^{p},
$$

and study these function for $m^{\prime}=1$ and also when $m=m^{\prime}+1$. In both cases they are rational functions in $x$; moreover in the first case they are related to the Chebyshev polynomial of the second kind as we now discuss. For $n \in \mathbb{Z}_{+}$define polynomials $a_{n}(x)$ by $a_{0}(x)=a_{1}(x)=1$ and for $n \geq 2$

$$
a_{n}(x)= \begin{cases}a_{n-1}(x)-x a_{n-2}(x) & \text { if } n \text { is odd } \\ (1+x) a_{n-1}(x)-x a_{n-2}(x) & \text { if } n \text { is even }\end{cases}
$$

We shall prove,

Theorem 2. For $s \in \mathbb{Z}_{+}$and $r \in\{0, \ldots, m-1\}$ we have

$$
A_{m s+r}^{1 \rightarrow m}(x)=\frac{a_{2 m-2 r-1}(x)}{\left(a_{m}(x) a_{m+1}(x)\right)^{s+1}},
$$

if $\left\lfloor\frac{m}{2}\right\rfloor \leq r \leq m-1$ and

$$
A_{m s+r}^{1 \rightarrow m}(x)=\frac{a_{m}(x) a_{m-2 r-1}(x)}{\left(a_{m}(x) a_{m+1}(x)\right)^{s+1}},
$$

if $0 \leq r \leq\left\lfloor\frac{m}{2}\right\rfloor-1$. 
Remark. The connection with Chebyshev polynomials is made as follows. Consider the following recurrences:

$$
U_{n+1}(x)=2 x U_{n}(x)-U_{n-1}(x), \quad U_{0}(x)=1, \quad U_{1}(x)=2 x .
$$

Let $P_{n}(x)$ be the polynomials defined by the recurrence

$$
P_{0}(x)=P_{1}(x)=1, P_{n+1}(x)=P_{n}(x)-x P_{n-1}(x) \text { for } n \geq 1 .
$$

It is known that the Chebyshev polynomials of the second kind satisfy the recurrences for $U$ and that $P_{n}\left(x^{2}\right)=x^{n} U_{n}\left((2 x)^{-1}\right)$. It is not hard to see that the polynomials $a_{n}(x)$ are given by

$$
a_{n}(x)=(1+x)^{\left\lfloor\frac{n}{2}\right\rfloor} P_{n}\left(\frac{x}{1+x}\right), n \geq 0 .
$$

Basically one just checks that the right hand side of the preceding equation satisfies the same recurrence relations as the $a_{n}(x)$. It is also useful to note here that $a_{2 n}(x)=a_{2 n-1}(x)-x^{2} a_{2 n-3}(x)$ and that $a_{n}(x)=(1-x) a_{n-2}(x)-x^{2} a_{n-4}(x)$ for $n \geq 4$.

\section{The Modules $V(\boldsymbol{\xi})$ And Dimension Bounds}

In this section we state the more general version of Theorem 1.

3.1. Let $\mathcal{P}_{\ell}$ be the set of all partitions $\boldsymbol{\xi}$ of length $\ell+1$ such that the following holds:

$$
\boldsymbol{\xi}=(\xi_{0} \geq \underbrace{(\xi+1) \geq \cdots \geq(\xi+1)}_{\ell-1-p} \geq \underbrace{\xi \geq \cdots \geq \xi}_{p} \geq \xi_{\ell}>0)
$$

where either $p=0$ and $\ell=1$ or $1 \leq p \leq \ell-1$ if $\ell>1$. For $\boldsymbol{\xi}=\left(\xi_{0} \geq \xi_{1} \geq \cdots \geq \xi_{\ell}\right) \in \mathcal{P}_{\ell}$, set

$$
|\boldsymbol{\xi}|=\sum_{j \geq 1} \xi_{j}, \quad \phi(\boldsymbol{\xi} ; k):= \begin{cases}\sum_{j \geq k+1} \xi_{j}-\frac{1}{2} \xi_{k+1}, & \text { if } 0 \leq k \leq \ell-2 \\ \left(\xi_{\ell}-\frac{1}{2} \xi_{\ell-1}\right)_{+}, & \text {if } k=\ell-1 \\ 0 & \text { else. }\end{cases}
$$

Define a partial order on $\mathcal{P}=\cup_{\ell \in \mathbb{N}} \mathcal{P}_{\ell}$ by: for $\boldsymbol{\xi}_{j} \in \mathcal{P}_{\ell_{j}}, j=1,2$, we say that

$$
\boldsymbol{\xi}_{1} \prec \boldsymbol{\xi}_{2} \Longleftrightarrow \text { either } \ell_{1}<\ell_{2} \text { or } \ell_{1}=\ell_{2} \text { and } \boldsymbol{\xi}_{1}<\boldsymbol{\xi}_{2} \text {, }
$$

where $<$ denotes the usual reverse lexicographic order on partitions.

3.2. We introduce the main objects of this paper.

Definition. Given $\boldsymbol{\xi} \in \mathcal{P}_{\ell}$ with $|\boldsymbol{\xi}|=n$, we define $V(\boldsymbol{\xi})$ to be the graded quotient of $D(1, n)$ by the submodule generated by the graded elements,

$$
\begin{aligned}
& y_{2 \ell} v_{n}, \quad y_{\ell+\frac{1}{2}} v_{n}, \\
& y_{2 \ell-2}^{\left(\xi_{\ell}+1\right)} v_{n}, \quad \text { if } \xi_{\ell}+1<\xi_{\ell-1}, \quad y_{\ell-\frac{1}{2}}^{\left(2 \phi \left(\boldsymbol{\xi}_{; \ell-1)+1)}\right.\right.} v_{n}, \quad \text { if } \quad \xi_{\ell}<\xi_{\ell-1} .
\end{aligned}
$$

If $n_{1} \in \mathbb{Z}$ with $n_{1} \geq-1$ and $n=n_{1} m+n_{0}, 0<n_{0} \leq m$ then we have an isomorphism of graded $\mathfrak{C} \mathfrak{g}$-modules

$$
V(\boldsymbol{\xi}) \cong D(m, n), \quad \boldsymbol{\xi}=(\underbrace{m \geq \cdots \geq m}_{n_{1}+1} \geq n_{0})
$$

Remark. Note that the definition of $V(\boldsymbol{\xi})$ is independent of $\xi_{0}$ unless $\ell=1$ and $2 \xi_{1}>\xi_{0}$. In what follows we denote by $v_{\boldsymbol{\xi}}$ the cyclic generator of $V(\boldsymbol{\xi})$. 
3.3. We now state the more general version of Theorem 1; the proof of this theorem can be found in the next section.

Theorem 3. For $\boldsymbol{\xi} \in \mathcal{P}_{\ell}$, we have an isomorphism of $\mathfrak{g}$-modules

$$
V(\boldsymbol{\xi}) \cong D\left(\xi_{1}, \xi_{1}\right)^{\otimes(\ell-1-p)} \otimes D\left(\xi_{\ell-1}, \xi_{\ell-1}\right)^{\otimes p} \otimes D\left(\xi_{\ell-1}, \xi_{\ell}\right)
$$

Further, the $\mathfrak{C} \mathfrak{g}$-module $V(\boldsymbol{\xi})$ admits a level $m$-Demazure flag if and only if $m \geq \xi_{0}$.

\section{Remark.}

(1) More generally the isomorphism in Theorem 3 is of $\mathfrak{C} \mathfrak{g}$-modules if we replace the tensor product by the fusion product; we refer the reader to [9] for the definition and properties of fusion products. Hence our result gives a presentation of a certain class of fusion products of different level Demazure modules.

(2) If $V(\boldsymbol{\xi})$ admits a level $m$ Demazure flag, then we have $m \geq \xi_{0}$. This implication can be proven similarly as in [6, Lemma 3.7] and the details will be omitted.

3.4. The modules $V(\boldsymbol{\xi}), \boldsymbol{\xi} \in \mathcal{P}_{\ell}$ admit another realization which we now discuss. We shall need some notation. For $s, r \in \mathbb{N}, k \in \mathbb{Z}_{+}$let

$$
\mathbf{S}_{\geq k}(r, s)=\left\{\mathbf{b}=\left(b_{j}\right)_{\substack{j \in \mathbb{Z}_{+} \\ j \geq k}}: b_{j} \in \mathbb{Z}_{+}, \quad \sum_{j \geq k} b_{j}=r, \quad \sum_{j \geq k} j b_{j}=s\right\},
$$

and for $(r, s) \in \mathbf{N}, \tilde{k} \in \mathbb{Z}_{+} / 2$ let

$$
\widetilde{\mathbf{S}}_{\geq \tilde{k}}(r, s)=\left\{\widetilde{\mathbf{b}}=\left(\tilde{b}_{j}\right)_{\substack{j j \in \mathbb{Z}_{+} \\ j \geq \tilde{k}}}: \tilde{b}_{j} \in \mathbb{Z}_{+}, \quad \sum_{j \geq \tilde{k}}\left(\tilde{b}_{j+1 / 2}+2 \tilde{b}_{j}\right)=2 r, \quad \sum_{j \geq \tilde{k}}\left(\left(j+\frac{1}{2}\right) \tilde{b}_{j+\frac{1}{2}}+2 j \tilde{b}_{j}\right)=s\right\} .
$$

The following elementary calculation will be used repeatedly.

Lemma. Let $(r, s) \in \mathbf{N}$, and $\widetilde{\mathbf{b}} \in \widetilde{\mathbf{S}}_{\geq k-\frac{3}{2}}(r, s)$ (resp. $\left.\widetilde{\mathbf{b}} \in \widetilde{\mathbf{S}}_{\geq k-\frac{5}{2}}(r, s)\right)$ such that $\tilde{b}_{j}=0$ for all $j \geq k$. Then we have

$$
\begin{gathered}
\tilde{b}_{k-\frac{1}{2}}+\tilde{b}_{k-1}=s-2 r(k-3 / 2), \quad \tilde{b}_{k-\frac{3}{2}}+\tilde{b}_{k-1}=2 r(k-1 / 2)-s \\
\left(\operatorname{resp} . \tilde{b}_{k-\frac{3}{2}}+2 \tilde{b}_{k-\frac{1}{2}}+\tilde{b}_{k-2}+3 \tilde{b}_{k-1}=s-2 r(k-5 / 2), \quad \tilde{b}_{k-\frac{5}{2}}-\tilde{b}_{k-\frac{1}{2}}+\tilde{b}_{k-2}-\tilde{b}_{k-1}=2 r(k-3 / 2)-s\right) .
\end{gathered}
$$

3.5. For any non-negative integer $b \in \mathbb{Z}_{+}$and $x \in \mathfrak{C g}$ set $x^{(b)}:=x^{b} / b$ !. Let $\mathbf{y}_{\geq k}(r, s), \widetilde{\mathbf{y}}_{\geq \tilde{k}}(r, s)$ be the following elements of $\mathbf{U}$ :

$$
\begin{aligned}
& \mathbf{y}_{\geq k}(r, s)=\sum_{\mathbf{b} \in \mathbf{S}_{\geq k}(r, s)} y_{2 k}^{\left(b_{k}\right)} y_{2 k+2}^{\left(b_{k+1}\right)} \cdots y_{2 s}^{\left(b_{s}\right)}, \\
& \widetilde{\mathbf{y}}_{\geq \tilde{k}}(r, s)=\sum_{\widetilde{\mathbf{b}} \in \widetilde{\mathbf{S}}_{\geq \tilde{k}}(r, s)} \prod_{n \geq \tilde{k}}\left(\widetilde{y}_{n+\frac{1}{2}}\right)^{\left(\tilde{b}_{n+\frac{1}{2}}\right)}\left(\widetilde{y}_{2 n}\right)^{\left(\tilde{b}_{n}\right)},
\end{aligned}
$$

where for $n \in \mathbb{Z}_{+}$we set

$$
2^{n} \widetilde{y}_{n+\frac{1}{2}}:=-y_{n+\frac{1}{2}} \quad 2^{2 n} \widetilde{y}_{2 n}:=\left((-1)^{n}-2\right) y_{2 n},
$$

and $\vec{\prod}_{n \geq 0}$ refers to the product of the specified factors written exactly in the increasing order of the indexing parameter. The following is proved along the same lines as Theorem 1 and Theorem 7 of $[14]$. 
Proposition. For $\boldsymbol{\xi} \in \mathcal{P}_{\ell}$ with $|\boldsymbol{\xi}|=n$, the module $V(\boldsymbol{\xi})$ is the quotient of $D(1, n)$ by the additional relations:

$$
\begin{aligned}
& \mathbf{y}_{\geq k}(r, s) v_{n}=0, \forall s, r \in \mathbb{N}, k \in \mathbb{Z}_{+} \text {with } \quad s+r \geq 1+k r+\sum_{j \geq k+1} \xi_{j}, \\
& \widetilde{\mathbf{y}}_{\geq k+\frac{1}{2}}(r, s) v_{n}=0, \forall(r, s) \in \mathbf{N}, k \in \mathbb{Z}_{+} \text {with } s \geq \frac{1}{2}+2 k r+\phi(\boldsymbol{\xi} ; k) .
\end{aligned}
$$

Moreover, there exists a surjective map of $\mathfrak{g}$-modules

$$
V(\boldsymbol{\xi}) \rightarrow D\left(\xi_{1}, \xi_{1}\right)^{\otimes(\ell-1-p)} \otimes D\left(\xi_{\ell-1}, \xi_{\ell-1}\right)^{\otimes p} \otimes D\left(\xi_{\ell-1}, \xi_{\ell}\right) .
$$

It is immediate from the proposition that

$$
y_{2 k}^{(r+1)} v_{\boldsymbol{\xi}}=0, \quad\left(\text { resp. } y_{k+\frac{1}{2}}^{(r+1)} v_{\boldsymbol{\xi}}=0\right)
$$

for all $r, k \in \mathbb{Z}_{+}$with $r \geq \sum_{j \geq k+1} \xi_{j}($ resp. $r \geq 2 \phi(\xi ; k))$.

\section{Demazure flags and Recursive formulae}

In this section, we prove Theorem 3 by an induction on $\ell$.

4.1. Recall from [14] that the Demazure module $D\left(\xi_{0}, \xi_{1}\right)$ is irreducible if and only if $2 \xi_{1} \leq \xi_{0}$ and otherwise decomposes into irreducible finite-dimensional $\mathfrak{g}$-modules as follows

$$
D\left(\xi_{0}, \xi_{1}\right) \cong \tau_{0}^{*} V\left(\xi_{1}\right) \oplus \cdots \oplus \tau_{2 \xi_{1}-\xi_{0}}^{*} V\left(\xi_{0}-\xi_{1}\right) .
$$

Hence

$$
\operatorname{dim} D\left(\xi_{0}, \xi_{1}\right)=\left(\xi_{1}+1\right)+\frac{1}{2}\left(\left(2 \xi_{1}-\xi_{0}\right)+\left(\xi_{0}+1\right)\right)
$$

Thus Proposition 3.5 gives a lower bound for the dimension of $V(\boldsymbol{\xi}), \boldsymbol{\xi} \in \mathcal{P}_{\ell}$ :

$$
\operatorname{dim} V(\boldsymbol{\xi}) \geq\left(\begin{array}{c}
\xi_{1}+2 \\
2
\end{array}\right)^{\ell-1-p}\left(\begin{array}{c}
\xi_{\ell-1}+2 \\
2
\end{array}\right)^{p}\left(\left(\xi_{\ell}+1\right)+\frac{1}{2}\left(\left(2 \xi_{1}-\xi_{\ell-1}\right)_{+}\left(\xi_{\ell-1}+1\right)\right)\right) .
$$

4.2. To see that induction begins at $\ell=1$ we write $\boldsymbol{\xi}=\left(\xi_{0}, \xi_{1}\right)$. Equation (3.5) implies that $V(\boldsymbol{\xi}) \cong D\left(\xi_{0}, \xi_{1}\right)$ and hence the first statement of Theorem 3 holds in this case. If $2 \xi_{1} \leq \xi_{0}$ or $\xi_{0}=m$ the second statement also holds since $D\left(\xi_{0}, \xi_{1}\right) \cong D\left(m, \xi_{1}\right)$. Otherwise consider the filtration of graded $\mathbf{U}$-modules

$$
0 \subset \mathbf{U} y_{\frac{1}{2}}^{2 \xi_{1}-\xi_{0}} v_{\boldsymbol{\xi}} \subset \mathbf{U} y_{\frac{1}{2}}^{2 \xi_{1}-\xi_{0}-1} v_{\boldsymbol{\xi}} \subset \cdots \subset \mathbf{U} y_{\frac{1}{2}}^{2 \xi_{1}-\xi_{0}-s} v_{\boldsymbol{\xi}} \subset V(\boldsymbol{\xi}),
$$

where $s=\left(2 \xi_{1}-\xi_{0}\right)-\left(2 \xi_{1}-m\right)_{+}-1$. Using Definition 3.2 it is easily seen that the successive quotients of the filtration in (4.2) are themselves quotients of a Demazure module of the form $\tau_{s}^{*} D\left(m, \xi_{1}-s\right)$. The dimension inequality in (4.1) now implies these maps are isomorphisms and hence $V(\boldsymbol{\xi})$ has a Demazure flag of level $m$ establishing the inductive step. Moreover we have also proved that

$$
\left[D\left(\xi_{0}, \xi_{1}\right), D(m, s)\right]_{q}= \begin{cases}q^{\xi_{1}-s}, & \text { if } s=\xi_{1} \text { or }\left(2 \xi_{1}-m\right)_{+}<\xi_{1}-s \leq\left(2 \xi_{1}-\xi_{0}\right)_{+} \\ 0, & \text { else. }\end{cases}
$$


4.3. For the rest of this section we fix an arbitrary $\boldsymbol{\xi} \in \mathcal{P}_{\ell}$ with $\ell>1$ and assume that the main theorem holds for all $\boldsymbol{\tau} \in \mathcal{P}$ with $\boldsymbol{\tau} \prec \boldsymbol{\xi}$. We further suppose $\xi_{0}=\xi_{1}$ (see Remark 3.2).

Define $\boldsymbol{\xi}^{+} \in \mathcal{P}$ by:

$$
\boldsymbol{\xi}^{+}:=\left((\xi+1)^{\ell-p+1}, \xi^{p-1}, \xi_{\ell}-1\right) .
$$

We have $\phi\left(\boldsymbol{\xi}^{+} ; \ell-1\right)=\left(\xi_{\ell}-\frac{1}{2} \xi_{\ell-1}-1-\delta_{p, 1} / 2\right)_{+}$. Noting that $\boldsymbol{\xi}^{+} \prec \boldsymbol{\xi}$, we have by the induction hypothesis that $V\left(\boldsymbol{\xi}^{+}\right)$admits a level $m$ Demazure flag iff $m \geq \xi+1$. We now prove,

Proposition. The assignment $v_{\boldsymbol{\xi}} \mapsto v_{\boldsymbol{\xi}^{+}}$defines a surjective morphism $\varphi^{+}: V(\boldsymbol{\xi}) \rightarrow V\left(\boldsymbol{\xi}^{+}\right) \rightarrow 0$ of $\mathfrak{C} \mathfrak{g}$-modules. Moreover $\operatorname{ker} \varphi^{+}$is generated by the elements,

$$
\begin{gathered}
y_{2 \ell-2}^{\left(\xi_{\ell}\right)} v_{\boldsymbol{\xi}}, \quad \delta_{p, 2} \delta_{\xi_{\ell}, 1} y_{\ell-\frac{3}{2}}^{\left(\xi_{\ell-1}\right)} v_{\boldsymbol{\xi}}, \quad \text { if } \quad 2 \xi_{\ell} \leq \xi_{\ell-1} \\
y_{\ell-\frac{3}{2}} v_{\boldsymbol{\xi}}, \quad \text { if } p=2, \quad \xi_{\ell}=1=\xi_{\ell-1},
\end{gathered}
$$

and in all other cases by $y_{\ell-\frac{1}{2}}^{\left(\left(2 \xi_{\ell-1}^{+}-\xi_{\ell-1}^{+}\right)_{+}^{+1)}\right.} v_{\boldsymbol{\xi}}$.

Proof. A simple checking shows that $v_{\boldsymbol{\xi}^{+}}$satisfies all the relations that $v_{\boldsymbol{\xi}}$ does and hence the existence of $\varphi^{+}$is immediate. It is also immediate that $\operatorname{ker} \varphi^{+}$is generated by the elements

$$
y_{2 \ell-2}^{\left(\xi_{\ell}\right)} v_{\boldsymbol{\xi}}, \quad y_{\ell-\frac{1}{2}}^{\left.\left(2 \xi_{\ell}^{+}-\xi_{\ell-1}^{+}\right)_{+}+1\right)} v_{\boldsymbol{\xi}}, \quad \delta_{p, 2} \delta_{\xi_{\ell}, 1} y_{\ell-\frac{3}{2}}^{\left(\xi_{\ell-1}\right)} v_{\boldsymbol{\xi}} .
$$

If $2 \xi_{\ell} \leq \xi_{\ell-1}$ the result follows since $y_{\ell-\frac{1}{2}} v_{\boldsymbol{\xi}}=0$. If $2 \xi_{\ell}>\xi_{\ell-1}$, we prove by a downward induction on $k$ that for all $0 \leq k \leq\left(2 \xi_{\ell}^{+}-\xi_{\ell-1}^{+}\right)_{+}$, we have

$$
y_{2 \ell-2}^{\left(\xi_{\ell}-k\right)} y_{\ell-\frac{1}{2}}^{(k)} v_{\boldsymbol{\xi}} \in \mathbf{U} y_{\ell-\frac{1}{2}}^{\left(\left(2 \xi_{\ell}^{+}-\xi_{\ell-1}^{+}\right)_{+}+1\right)} v_{\boldsymbol{\xi}}
$$

Assuming we have done this, notice that by taking $k=0$ the proof is complete unless we are in the case of $p=2$ and $\xi_{\ell}=\xi_{\ell-1}=1$ when we also have to prove that

$$
y_{\ell-\frac{1}{2}} v_{\boldsymbol{\xi}} \in \mathbf{U} y_{\ell-\frac{3}{2}} v_{\boldsymbol{\xi}}
$$

But this is immediate by applying an element of $\mathfrak{C h}_{+}$of appropriate grade. We set $2 r=2 \xi_{\ell}-k$ and $s=2(\ell-1) \xi_{\ell}-\left(\ell-\frac{3}{2}\right) k$. Note that

$$
s \geq \frac{1}{2}+\left(2 \xi_{\ell}-k\right)(\ell-2)+\frac{1}{2} \xi_{\ell-1}+\xi_{\ell}=\frac{1}{2}+2 r(\ell-2)+\phi(\boldsymbol{\xi}, \ell-2) .
$$

For an arbitrary element $\widetilde{\mathbf{b}} \in \widetilde{\mathbf{S}}_{\geq \ell-\frac{3}{2}}(r, s)$ with $\tilde{b}_{j}=0$ for $j \geq \ell$ we get with Lemma 3.4

$$
\tilde{b}_{\ell-\frac{1}{2}}+\tilde{b}_{\ell-1}=\xi_{\ell}, \quad \tilde{b}_{\ell-\frac{3}{2}}+\tilde{b}_{\ell-1}=\xi_{\ell}-k,
$$

which implies $\tilde{b}_{\ell-\frac{3}{2}}=\tilde{b}_{\ell-\frac{1}{2}}-k \geq 0$. Hence, together with (3.9) and (4.6) we get

$$
y_{2 \ell-2}^{\left(\xi_{\ell}-k\right)} y_{\ell-\frac{1}{2}}^{(k)} v_{\boldsymbol{\xi}} \in \sum_{t>k} \mathbf{U} y_{2 \ell-2}^{\left(\xi_{\ell}-t\right)} y_{\ell-\frac{1}{2}}^{(t)} v_{\boldsymbol{\xi}}
$$

If $k=\left(2 \xi_{\ell}^{+}-\xi_{\ell-1}^{+}\right)_{+}$equation (4.5) is immediate with (4.7). Otherwise we know by the induction hypothesis that each summand in (4.7) has the desired property.

In the rest of this section we shall show by doing a case by case analysis that $\operatorname{ker} \varphi^{+}$has a filtration such that the successive quotients are of the form $V\left(\boldsymbol{\xi}^{\prime}\right)$, with $\boldsymbol{\xi}^{\prime} \prec \boldsymbol{\xi}$. Since the induction hypothesis applies to the $\boldsymbol{\xi}^{\prime}$ it follows that $\operatorname{ker} \varphi^{+}$has a level $m$ Demazure flag and also allows us to get an upper bound for $V(\boldsymbol{\xi})$; together with the lower bound established in Proposition 3.5 we then complete the inductive step. 
4.4. Given $\boldsymbol{\xi}=\left((\xi+1)^{\ell-p}, \xi^{p}, \xi_{\ell}\right) \in \mathcal{P}_{\ell}$, set

$$
k(\boldsymbol{\xi})= \begin{cases}2, & \text { if } 2 \xi_{\ell}-\xi_{\ell-1} \geq 3 \text { and } p=1 \\ 1, & \text { if } 2 \xi_{\ell}-\xi_{\ell-1}=2 \text { or } 2 \xi_{\ell}-\xi_{\ell-1} \geq 3 \text { and } p>1 \\ 0, & \text { if } 2 \xi_{\ell}-\xi_{\ell-1}=1 \\ -1, & \text { if } 2 \xi_{\ell}-\xi_{\ell-1} \leq 0\end{cases}
$$

Equivalently,

$$
k(\boldsymbol{\xi})=\left(2 \xi_{\ell}-\xi_{\ell-1}\right)_{+}-\left(2 \xi_{\ell}^{+}-\xi_{\ell-1}^{+}\right)_{+}-1 .
$$

For $-1 \leq j \leq k(\boldsymbol{\xi})$, define partitions $\boldsymbol{\xi}(j)$ as follows:

$$
\boldsymbol{\xi}(j)=\left((\xi+1)^{\ell-p+1-\delta_{j, 0}-\delta_{j,-1}}, \xi^{p-1+\delta_{j, 0}}, \xi_{\ell-1}-\xi_{\ell}+\delta_{j, 2}\right) .
$$

It is easily seen that $\boldsymbol{\xi}(j) \in \mathcal{P}$ and $\boldsymbol{\xi}(j) \prec \boldsymbol{\xi}$ for $-1 \leq j \leq k(\boldsymbol{\xi})$.

4.5. We analyze $\operatorname{ker} \varphi^{+}$under the assumption that $2 \xi_{\ell}>\xi_{\ell-1}$.

Proposition. Suppose that $2 \xi_{\ell}>\xi_{\ell-1}$.

(i) Let $\delta_{\xi_{\ell}, \xi_{\ell-1}} \delta_{p, 2}=0$. For $-1 \leq j \leq k(\boldsymbol{\xi})$ there exists graded $\mathfrak{C} \mathfrak{g}$-submodules $V_{j} \subset \operatorname{ker} \varphi^{+}$with

$$
\begin{gathered}
V_{-1} \cong \delta_{\xi_{\ell} \xi_{\ell-1}} \delta_{p, 1} \tau_{4 \xi_{\ell}(\ell-1)}^{*} V(\boldsymbol{\xi}(-1)), \quad V_{k(\boldsymbol{\xi})}=\operatorname{ker} \varphi^{+}, \\
V_{j-1} \subset V_{j}, \quad V_{j} / V_{j-1} \cong \tau_{(2 \ell-1)\left(2 \xi_{\ell}-\xi_{\ell-1}-j\right)}^{*} V(\boldsymbol{\xi}(j)), \quad 0 \leq j \leq k(\boldsymbol{\xi}) .
\end{gathered}
$$

(ii) Let $\delta_{\xi_{\ell}, \xi_{\ell-1}} \delta_{p, 2}=1$. If $\xi_{\ell}=1$ we have a short exact sequence of graded $\mathfrak{C} \mathfrak{g}$-modules

$$
0 \rightarrow \tau_{(2 \ell-1)}^{*} V(\boldsymbol{\xi}(0)) \rightarrow \operatorname{ker} \varphi_{+} \rightarrow \tau_{(2 \ell-3)}^{*} V\left(\left(\xi_{\ell-1}+1\right)^{\ell-1}\right) \rightarrow 0 .
$$

If $\xi_{\ell}>1$ then there exists graded $\mathfrak{C} \mathfrak{g}$-submodules $V_{0} \subset V_{1}$ of $\operatorname{ker} \varphi^{+}$such that

$$
\begin{gathered}
V_{0} \cong \tau_{\xi_{\ell}(2 \ell-1)}^{*} V(\boldsymbol{\xi}(0)), \quad V_{1} / V_{0} \cong \tau_{4 \xi_{\ell}(\ell-1)-(2 \ell-1)}^{*} V\left(\left(\xi_{\ell-1}+1\right)^{\ell-1}\right), \\
\operatorname{ker} \varphi^{+} / V_{1} \cong \tau_{\left(\xi_{\ell}-1\right)(2 \ell-1)}^{*} V(\boldsymbol{\xi}(1)) .
\end{gathered}
$$

4.6. To complete our analysis of $\operatorname{ker} \varphi^{+}$we shall prove,

Proposition. Assume that $2 \xi_{\ell} \leq \xi_{\ell-1}$.

(i) Suppose that $\delta_{\xi_{\ell}, 1} \delta_{p, 2}=0$ and $\left(1-\delta_{2 \xi_{\ell}, \xi_{\ell-1}}\right) \delta_{p, 1}=0$. Then we have an isomorphism of graded $\mathfrak{C} \mathfrak{g}$-modules

$$
\tau_{4(\ell-1) \xi_{\ell}}^{*} V(\boldsymbol{\xi}(-1)) \cong \operatorname{ker} \varphi^{+} .
$$

(ii) Suppose that $\delta_{\xi_{\ell}, 1} \delta_{p, 2}=0$ and $\left(1-\delta_{2 \xi_{\ell}, \xi_{\ell-1}}\right) \delta_{p, 1}=1$. We have a short exact sequence of graded $\mathfrak{C} \mathfrak{g}$-modules

$$
0 \rightarrow \tau_{(2 \ell-3) \xi_{\ell-1}+2 \xi_{\ell}}^{*} V\left(\left(\xi_{\ell-1}+1\right)^{\ell-1}, \xi_{\ell}\right) \rightarrow \operatorname{ker} \varphi^{+} \rightarrow \tau_{4(\ell-1) \xi_{\ell}}^{*} V(\boldsymbol{\xi}(-1)) \rightarrow 0 .
$$

(iii) Suppose that $\delta_{\xi_{\ell}, 1} \delta_{p, 2}=1$. We have a short exact sequence of graded $\mathfrak{C g}$-modules

$$
0 \rightarrow \tau_{4(\ell-1)}^{*} V(\boldsymbol{\xi}(-1)) \rightarrow \operatorname{ker} \varphi^{+} \rightarrow \tau_{\xi_{\ell-1}(2 \ell-3)}^{*} V\left(\left(\xi_{\ell-1}+1\right)^{\ell-1}\right) \rightarrow 0
$$


4.7. Before we prove Proposition 4.5 and Proposition 4.6 we show how we can use both to prove Theorem 3. We obtain a filtration of $\operatorname{ker} \varphi^{+}$by graded $\mathfrak{C} \mathfrak{g}$-submodules such that the successive quotients are of the form $V\left(\boldsymbol{\xi}^{\prime}\right)$, with $\boldsymbol{\xi}^{\prime} \prec \boldsymbol{\xi}$. By applying the induction hypothesis to each $V\left(\boldsymbol{\xi}^{\prime}\right)$ and $V\left(\boldsymbol{\xi}^{+}\right)$we obtain that $V(\boldsymbol{\xi})$ admits a level $m$ Demazure flag for all $m \geq \xi+1$. Hence the second part of Theorem 3 is proven unless we are in the case $\xi_{0}=\xi=m$ when we also have to prove that $V(\boldsymbol{\xi})$ has a level $\xi_{0}$ Demazure flag. But this is immediate with (3.5) since $V(\boldsymbol{\xi})$ itself is a Demazure module of level $\xi_{0}$.

Recall the dimension bound from (4.1). Now we also have an upper bound using the above filtration. A long but tedious calculation shows that these bounds coincide and hence we have equality in (4.1). This proves the first part of the theorem together with Proposition 3.5. Moreover, the explicit construction of the filtration yields the following recursive formulae:

Corollary. Let $\boldsymbol{\xi} \in \mathcal{P}_{\ell}, m \in \mathbb{N}$ such that $m \geq \xi_{0}$ and $D$ a level $m$ Demazure module.

(1) If $2 \xi_{\ell}>\xi_{\ell-1}$ we have

$$
\begin{aligned}
{[V(\boldsymbol{\xi}): D]_{q} } & =\left[V\left(\boldsymbol{\xi}^{+}\right): D\right]_{q}+\sum_{j=0}^{k(\boldsymbol{\xi})} q^{(2 \ell-1)\left(2 \xi_{\ell}-\xi_{\ell-1}-j\right)}[V(\boldsymbol{\xi}(j)): D]_{q} \\
& +\delta_{\xi_{\ell}, \xi_{\ell-1}}\left(\delta_{p, 1}+\delta_{p, 2}\right) q^{4 \xi_{\ell}(\ell-1)-(p-1)(2 \ell-1)}\left[V\left(\left(\xi_{\ell-1}+1\right)^{\ell-1}\right): D\right]_{q} .
\end{aligned}
$$

(2) If $2 \xi_{\ell} \leq \xi_{\ell-1}$ we have

$$
\begin{aligned}
{[V(\boldsymbol{\xi}): D]_{q} } & =\left[V\left(\boldsymbol{\xi}^{+}\right): D\right]_{q}+q^{4 \xi_{\ell}(\ell-1)}[V(\boldsymbol{\xi}(-1)): D]_{q} \\
& +q^{(2 \ell-3) \xi_{\ell-1}+2 \xi_{\ell}}\left(1-\delta_{2 \xi_{\ell}, \xi_{\ell-1}}\right) \delta_{p, 1}\left[V\left(\left(\xi_{\ell-1}+1\right)^{\ell-1}, \xi_{\ell}\right): D\right]_{q} \\
& +q^{(2 \ell-3) \xi_{\ell-1}} \delta_{\xi_{\ell}, 1} \delta_{p, 2}\left[V\left(\left(\xi_{\ell-1}+1\right)^{\ell-1}\right): D\right]_{q}
\end{aligned}
$$

4.8. We need the following technical lemma whose proof we postpone to the end of the section.

Lemma. We have the following relations in $V(\boldsymbol{\xi})$ :

(i)

(ii) If $2 \xi_{\ell} \leq \xi_{\ell-1}$, we have

$$
y_{2 \ell-4}^{\left(\xi_{\ell-1}-\xi_{\ell}+1\right)} y_{2 \ell-2}^{\left(\xi_{\ell}\right)} v_{\boldsymbol{\xi}}=0
$$

$$
y_{\ell-\frac{3}{2}}^{\left(\xi_{\ell-2}-\xi_{\ell}+1\right)} y_{2 \ell-2}^{\left(\xi_{\ell}\right)} v_{\boldsymbol{\xi}}=0=y_{2 \ell-4}^{\left(\xi_{\ell}+1\right)} y_{\ell-\frac{3}{2}}^{\left(\xi_{\ell-1}-2 \xi_{\ell}\right)} y_{2 \ell-2}^{\left(\xi_{\ell}\right)} v_{\boldsymbol{\xi}}
$$

(iii) If $\xi_{\ell}=\xi_{\ell-1}$ we have

$$
y_{\ell-\frac{3}{2}}^{\left(\xi_{\ell}+1\right)} y_{\ell-\frac{1}{2}}^{\left(\xi_{\ell}\right)} v_{\boldsymbol{\xi}}=0, \quad y_{\ell-\frac{3}{2}}^{\left(\xi_{\ell}\right)} y_{\ell-\frac{1}{2}}^{\left(\xi_{\ell}\right)} v_{\boldsymbol{\xi}} \in \mathbf{U}^{-} y_{2 \ell-2}^{\left(\xi_{\ell}\right)} v_{\boldsymbol{\xi}}
$$

(iv) If $\xi_{\ell}=\xi_{\ell-1}=\xi_{\ell-2}$ we have

$$
\left\{y_{\ell-\frac{3}{2}}^{\left(\xi_{\ell}+1\right)} y_{\ell-\frac{1}{2}}^{\left(\xi_{\ell}-1\right)} v_{\boldsymbol{\xi}}, \quad y_{2 \ell-4} y_{\ell-\frac{3}{2} \delta}^{\left(\xi_{\ell}\right)} y_{\ell-\frac{1}{2}}^{\left(\xi_{\ell}-1\right)} v_{\boldsymbol{\xi}}\right\} \subset \mathbf{U}^{-} y_{\ell-\frac{1}{2}}^{\left(\xi_{\ell}\right)} v_{\boldsymbol{\xi}} .
$$

4.9. Proof of Proposition 4.5(i). Suppose that $2 \xi_{\ell}>\xi_{\ell-1}$ and $\delta_{\xi_{\ell}, \xi_{\ell-1}} \delta_{p, 2}=0$. Set

$$
w_{-1}=\delta_{\xi_{\ell} \xi_{\ell-1}} \delta_{p, 1} y_{\ell-\frac{3}{2}}^{\left(\xi_{\ell}\right)} y_{\ell-\frac{1}{2}}^{\left(\xi_{\ell}\right)} v_{\boldsymbol{\xi}}, \quad w_{j}=y_{\ell-\frac{1}{2}}^{\left(2 \xi_{\ell}-\xi_{\ell-1}-j\right)} v_{\boldsymbol{\xi}}, \quad 0 \leq j \leq k(\boldsymbol{\xi}) .
$$

Let $V_{j}$ be the $\mathfrak{C} \mathfrak{g}$-submodule of $V(\boldsymbol{\xi})$ generated by $w_{j}$ for $-1 \leq j \leq k(\boldsymbol{\xi})$ and note that $V_{k(\boldsymbol{\xi})}=\operatorname{ker} \varphi^{+}$ by Proposition 4.3 and (4.8). An easy calculation show that we have a surjective map from the appropriate level one Demazure module onto $V_{j} / V_{j-1}, 0 \leq j \leq k(\boldsymbol{\xi})$. We will further show that the 
highest weight vector $\bar{w}_{j}$ in $V_{j} / V_{j-1}$ satisfies the defining relations of $V(\boldsymbol{\xi}(j))$. It means, we shall prove that

$$
\begin{aligned}
& y_{2 \ell} \bar{w}_{j}=y_{\ell+\frac{1}{2}} \bar{w}_{j}=y_{\ell-\frac{1}{2}} \bar{w}_{j}=0, \\
& y_{2 \ell-2}^{\left(\xi_{\ell-1}-\xi_{\ell}+\delta_{j, 2}+1\right)} \bar{w}_{j}=\delta_{\xi_{\ell}, \xi_{\ell-1}} \delta_{p, 1} \delta_{j, 0} y_{\ell-\frac{3}{2}}^{\left(\xi_{\ell}\right)} \bar{w}_{j}=0 .
\end{aligned}
$$

The relations in (4.9) are obviously satsified since $\left[y_{2 \ell}, y_{\ell-\frac{1}{2}}\right]=0,\left[y_{\ell+\frac{1}{2}}, y_{\ell-\frac{1}{2}}\right]=y_{2 \ell}$, and

$$
y_{\ell-\frac{1}{2}}^{\left(2 \xi_{\ell}-\xi_{\ell-1}+1\right)} v_{\boldsymbol{\xi}}=y_{\ell+\frac{1}{2}} v_{\boldsymbol{\xi}}=y_{2 \ell} v_{\boldsymbol{\xi}}=0
$$

by (3.10). Now we turn our attention to the relations in (4.10). By construction we have

$$
\delta_{\xi_{\ell}, \xi_{\ell-1}} \delta_{p, 1} y_{\ell-\frac{3}{2}}^{\left(\xi_{\ell}\right)} w_{0}=w_{-1}=0 .
$$

Hence it remains to show

$$
y_{2 \ell-2}^{\left(\xi_{\ell-1}-\xi_{\ell}+\delta_{j, 2}+1\right)} w_{j} \in \mathbf{U} w_{j-1} .
$$

Set

$$
r=\frac{1}{2} \xi_{\ell-1}+1-\frac{\delta_{j, 1}}{2} \text { and } s=\left(\ell-\frac{1}{2}\right)\left(2 \xi_{\ell}-\xi_{\ell-1}-j\right)+2(\ell-1)\left(\xi_{\ell-1}-\xi_{\ell}+\delta_{j, 2}+1\right) .
$$

Similarly as in the proof of Proposition 4.3 we can show that each $\widetilde{\mathbf{b}} \in \widetilde{\mathbf{S}}_{\geq \ell-\frac{3}{2}}(r, s)$ with $\tilde{b}_{i}=0$ for $i \geq \ell$ satisfies $\tilde{b}_{\ell-\frac{1}{2}} \geq 2 \xi_{\ell}-\xi_{\ell-1}-j$. Together with Proposition 3.5 and

$$
s \geq \frac{1}{2}+2 r(\ell-2)+\phi(\boldsymbol{\xi}, \ell-2)
$$

we get

$$
\widetilde{y}_{2 \ell-2}^{\left(\xi_{\ell-1}-\xi_{\ell}+\delta_{j, 2}+1\right)} \widetilde{y}_{\ell-\frac{1}{2}}^{\left(2 \xi_{\ell}-\xi_{\ell-1}-j\right)} v_{\boldsymbol{\xi}} \in \sum_{t>2 \xi_{\ell}-\xi_{\ell-1}-j} \mathbf{U} \widetilde{y}_{\ell-\frac{1}{2}}^{(t)} v_{\boldsymbol{\xi}}
$$

which implies (4.11). Thus we get surjective maps

$$
\tau_{(2 \ell-1)\left(2 \xi_{\ell}-\xi_{\ell-1}-j\right)}^{*} V(\boldsymbol{\xi}(j)) \rightarrow V_{j} / V_{j-1}, \quad 0 \leq j \leq k(\boldsymbol{\xi}) .
$$

Moreover, we note that Lemma 4.8 (i) and (iii) imply that we also have a surjective map

$$
\delta_{\xi_{\ell}, \xi_{\ell-1}} \delta_{p, 1} \tau_{4 \xi_{\ell}(\ell-1)}^{*} V(\boldsymbol{\xi}(-1)) \rightarrow V_{-1} .
$$

The proof is complete if we show that the maps in (4.12) and (4.13) are isomorphisms. Applying our induction hypothesis, we obtain an upper bound for $\operatorname{dim} V(\boldsymbol{\xi})$ by (4.12) and (4.13). A straightforward calculation shows that the lower bound established in (4.1) coincides with this upper bound, which proves the proposition.

4.10. Proof of Proposition 4.5(ii). Assume that $2 \xi_{\ell}>\xi_{\ell-1}$ and $\delta_{\xi_{\ell}, \xi_{\ell-1}} \delta_{p, 2}=1$. Set

$$
w_{0}=y_{\left(\ell-\frac{1}{2}\right)}^{\left(\xi_{\ell}\right)} v_{\boldsymbol{\xi}}, \quad w_{1}=y_{\ell-\frac{3}{2}}^{\left(\xi_{\ell}\right)} y_{\ell-\frac{1}{2}}^{\left(\xi_{\ell}-1\right)} v_{\boldsymbol{\xi}}, \quad w_{2}=\left(1-\delta_{\xi_{\ell}, 1}\right) y_{\ell-\frac{1}{2}}^{\left(\xi_{\ell}-1\right)} v_{\boldsymbol{\xi}}
$$

and let $V_{j}$ be the submodule generated by the elements $w_{i}, 0 \leq i \leq j$. Note that $V_{2}=\operatorname{ker} \varphi^{+}$by Proposition 4.3 and if $\xi_{\ell}=1$ we have $V_{1}=V_{2}=\operatorname{ker} \varphi^{+}$.

The idea is again to show that there is a cyclic generator satisfying the defining relations of $V(\boldsymbol{\xi}(j))$ and $V\left(\left(\xi_{\ell-1}+1\right)^{\ell-1}\right)$ respectively and to use the dimension bound given in (4.1). The harder relations are stated in Lemma 4.8 and all other relations are easy to check. To be more precise, in addition to the relations proven in Lemma 4.8 (iii)-(iv) we need to verify

$$
y_{2 \ell-2} y_{\ell-\frac{1}{2}}^{\left(\xi_{\ell}-1\right)} v_{\boldsymbol{\xi}} \in \mathbf{U}^{-} y_{\ell-\frac{1}{2}}^{\left(\xi_{\ell}\right)} v_{\boldsymbol{\xi}}, \quad y_{2 \ell-2} y_{\ell-\frac{1}{2}}^{\left(\xi_{\ell}\right)} v_{\boldsymbol{\xi}}=0
$$


The proof is similar and will be omitted.

4.11. Proof of Proposition 4.6(i). Assume that $2 \xi_{\ell} \leq \xi_{\ell-1}, \delta_{p, 2} \delta_{\xi_{\ell}, 1}=0$ and $\left(1-\delta_{2 \xi_{\ell}, \xi_{\ell-1}}\right) \delta_{p, 1}=$ 0 . By Lemma 4.8 and (3.10) we know that $y_{2 \ell-2}^{\left(\xi_{\ell}\right)} v_{\boldsymbol{\xi}}$, which is the generator of the kernel by Proposition 4.3, satisfies the defining relations of $\tau_{4(\ell-1) \xi_{\ell}}^{*} V(\boldsymbol{\xi}(-1))$ given in Definition 3.2. Again, a simple dimension argument using our induction hypothesis and (4.1) finishes the proof.

4.12. Proof of Proposition 4.6(ii). We consider the case $2 \xi_{\ell} \leq \xi_{\ell-1}, \delta_{p, 2} \delta_{\xi_{\ell}, 1}=0$ and (1$\left.\delta_{2 \xi_{\ell}, \xi_{\ell-1}}\right) \delta_{p, 1}=1$. Set

$$
w_{0}=y_{\ell-\frac{3}{2}}^{\left(\xi_{\ell-1}-2 \xi_{\ell}\right)} y_{2 \ell-2}^{\left(\xi_{\ell}\right)} v_{\boldsymbol{\xi}}, \quad w_{1}=y_{2 \ell-2}^{\left(\xi_{\ell}\right)} v_{\boldsymbol{\xi}},
$$

and let $V_{j}$ be the submodule generated by the element $w_{j}, 0 \leq j \leq 1$. Note that Proposition 4.3 implies $V_{1}=\operatorname{ker} \varphi^{+}$. From Lemma 4.8 and (3.10) we obtain the following surjective maps

$$
\tau_{4(\ell-1) \xi_{\ell}}^{*} V(\boldsymbol{\xi}(-1)) \rightarrow V_{1} / V_{0}
$$

and

$$
\tau_{(2 \ell-3) \xi_{\ell-1}+2 \xi_{\ell}}^{*} V\left(\left(\xi_{\ell-1}+1\right)^{\ell-1}, \xi_{\ell}\right) \rightarrow V_{0} .
$$

Again a simple dimension argument shows that the maps in (4.14) and (4.15) are isomorphisms.

4.13. Proof of Proposition 4.6(iii). We consider the remaining case $2 \xi_{\ell} \leq \xi_{\ell-1}$ and $\delta_{p, 2} \delta_{\xi_{\ell}, 1}=$ 1. Set

$$
w_{0}=y_{2 \ell-2} v_{\boldsymbol{\xi}}, \quad w_{1}=y_{\ell-\frac{3}{2}}^{\left(\xi_{\ell-1}\right)} v_{\boldsymbol{\xi}}
$$

and let $V_{j}$ be the submodule generated by the element $w_{i}, 0 \leq i \leq j$. Note that $V_{1}=\operatorname{ker} \varphi^{+}$. Lemma 4.8 immediately implies

$$
\tau_{4(\ell-1)}^{*} V(\boldsymbol{\xi}(-1)) \rightarrow V_{0} .
$$

Again by a dimension argument it remains to show that the highest weight vector in $V_{1} / V_{0}$ satisfies

$$
y_{2(\ell-2)} y_{\ell-\frac{3}{2}}^{\left(\xi_{\ell-1}\right)} v_{\boldsymbol{\xi}}=y_{\ell-\frac{3}{2}}^{\left(\xi_{\ell-1}+1\right)} v_{\boldsymbol{\xi}}=0 .
$$

Let $k=(\ell-3), 2 r=\xi_{\ell-1}+1$ and $s=\left(\ell-\frac{3}{2}\right)\left(\xi_{\ell-1}+1\right)$. We obviously have $s \geq \frac{1}{2}+2 r(\ell-3)+\phi(\boldsymbol{\xi}, \ell-3)$ and hence $\widetilde{\mathbf{y}}_{\geq \ell-\frac{5}{2}}(r, s) v_{\boldsymbol{\xi}}=0$. Our aim is to prove that

$$
\widetilde{\mathbf{y}}_{\geq \ell-\frac{5}{2}}(r, s) v_{\boldsymbol{\xi}}=y_{\ell-\frac{3}{2}}^{\left(\xi_{\ell-1}+1\right)} v_{\boldsymbol{\xi}}+V_{0}
$$

or equivalently

$$
\widetilde{\mathbf{b}} \in \widetilde{\mathbf{S}}_{\geq \ell-\frac{3}{2}}(r, s) \Longrightarrow \tilde{b}_{\ell-1}>0 \text { or } \tilde{b}_{\ell-\frac{3}{2}}=\xi_{\ell-1}+1 .
$$

Assume $\tilde{b}_{\ell-1}=0$. We have with Lemma 3.4

$$
\tilde{b}_{\ell-\frac{3}{2}}+\tilde{b}_{\ell-2}=\xi_{\ell-1}+1, \quad \tilde{b}_{\ell-\frac{5}{2}}+\tilde{b}_{\ell-2}=0
$$

which proves (4.16). Now we set $2 r=\xi_{\ell-1}+2, s=\left(\ell-\frac{3}{2}\right) \xi_{\ell-1}+2(\ell-2)$ and obtain with similar calculations as above that any $\widetilde{\mathbf{b}} \in \widetilde{\mathbf{S}}_{\geq \ell-\frac{5}{2}}(r, s)$ with $\tilde{b}_{i}=0$ for $i \geq \ell-1$ is of the form

$$
\tilde{b}_{\ell-2}=1, \tilde{b}_{\ell-\frac{3}{2}}=\xi_{\ell-1}, \tilde{b}_{\ell-1}=\tilde{b}_{\ell-\frac{5}{2}}=0 \quad \text { or } \quad \tilde{b}_{\ell-2}=\tilde{b}_{\ell-1}=0, \tilde{b}_{\ell-\frac{3}{2}}=\xi_{\ell-1}+1, \tilde{b}_{\ell-\frac{5}{2}}=1 .
$$

Now (4.16) finishes the proof. 
4.14. Proof of Lemma 4.8. Proof of part (i): Let $r=1+\xi_{\ell-1}, s=\xi_{\ell}+(\ell-2)\left(\xi_{\ell-1}+1\right)$. We have

$$
r+s \geq 1+r(\ell-2)+\xi_{\ell-1}+\xi_{\ell}
$$

which implies together with $(3.8)$

$$
\mathbf{y}_{\geq \ell-2}(r, s) v_{\boldsymbol{\xi}}=y_{2 \ell-4}^{\left(\xi_{\ell-1}-\xi_{\ell}+1\right)} y_{2 \ell-2}^{\left(\xi_{\ell}\right)} v_{\boldsymbol{\xi}}=0 .
$$

Proof of part (ii): We fix $t \in\left\{0, \ldots, \xi_{\ell}\right\}$ and set $2 r=2 t+\xi_{\ell-1}+1, s=\xi_{\ell}+\left(\ell-\frac{3}{2}\right)\left(\xi_{\ell-1}+1\right)+2(\ell-2) t$. We obtain

$$
s \geq \frac{1}{2}+\left(2 t+\xi_{\ell-1}+1\right)(\ell-2)+\frac{1}{2} \xi_{\ell-1}+\xi_{\ell}=\frac{1}{2}+2 r(\ell-2)+\phi(\xi, \ell-2)
$$

and hence with (3.9) we get

$$
\widetilde{\mathbf{y}}_{\geq \ell-\frac{3}{2}}(r, s) v_{\boldsymbol{\xi}}=\widetilde{y}_{\ell-\frac{3}{2}}^{\left(\xi_{\ell-1}-2 \xi_{\ell}+4 t+1\right)} \widetilde{y}_{2 \ell-2}^{\left(\xi_{\ell}-t\right)} v_{\boldsymbol{\xi}}=0
$$

which proves the first part. In order to prove the second part we fix $z \in\left\{1, \ldots, \xi_{\ell}+1\right\}$ and set $2 r=\xi_{\ell-1}+2 z, s=\xi_{\ell}+\xi_{\ell-1}\left(\ell-\frac{3}{2}\right)+2(\ell-2) z$. If $\ell=2$ the statement is obvious; so let $\ell \geq 3$. We have

$$
s \geq \frac{1}{2}+2 r(\ell-3)+\phi(\boldsymbol{\xi}, \ell-3)
$$

and therefore $\widetilde{\mathbf{y}}_{\geq \ell-\frac{5}{2}}(r, s) v_{\boldsymbol{\xi}}=0$. Let $\widetilde{\mathbf{b}} \in \widetilde{\mathbf{S}}_{\geq \ell-\frac{5}{2}}(r, s)$ with $\tilde{b}_{j}=0$ for all $j \geq \ell-\frac{1}{2}$ and set $\tilde{b}_{\ell-1}=\xi_{\ell}-t$ for some $t \in\left\{0, \ldots, \xi_{\ell}\right\}$. From Lemma 3.4 we get

$$
s-r(2 \ell-5)=\left(\tilde{b}_{\ell-\frac{3}{2}}+3 \tilde{b}_{\ell-1}+\tilde{b}_{\ell-2}\right)=\xi_{\ell-1}+\xi_{\ell}+z
$$

which implies

$$
z-\xi_{\ell}=2 r-\left(\xi_{\ell-1}+\xi_{\ell}+z\right)=\left(\tilde{b}_{\ell-\frac{5}{2}}+\tilde{b}_{\ell-2}-\tilde{b}_{\ell-1}\right), \quad \tilde{b}_{\ell-\frac{5}{2}}+\tilde{b}_{\ell-2}=z-t .
$$

Thus $\tilde{b}_{\ell-2} \leq z-t$ and with (4.18) we obtain

$$
\tilde{b}_{\ell-\frac{3}{2}} \geq \xi_{\ell-1}-2 \xi_{\ell}+4 t
$$

Now we use (4.17) and obtain that $\widetilde{\mathbf{b}}$ must be of the form

$$
\tilde{b}_{\ell-1}=\xi_{\ell}-t, \tilde{b}_{\ell-\frac{3}{2}}=\xi_{\ell-1}-2 \xi_{\ell}+4 t, \tilde{b}_{\ell-2}=z-t, \tilde{b}_{\ell-\frac{5}{2}}=0, \text { for some } t \in\left\{0, \ldots, \xi_{\ell}\right\} .
$$

Therefore

$$
\widetilde{\mathbf{y}}_{\geq \ell-\frac{5}{2}}(r, s) v_{\boldsymbol{\xi}}=\sum_{t=0}^{\min \left\{z, \xi_{\ell}\right\}} \widetilde{y}_{\ell-\frac{3}{2}}^{\left(\xi_{\ell-1}-2 \xi_{\ell}+4 t\right)} \widetilde{y}_{2 \ell-4}^{(z-t)} \widetilde{y}_{2 \ell-2}^{\left(\xi_{\ell}-t\right)} v_{\boldsymbol{\xi}}=0 .
$$

Acting with $\widetilde{y}_{2 \ell-4}^{\left(\xi_{\ell}+1-y\right)}$ on (4.19) yields

$$
\sum_{t=0}^{\min \left\{z, \xi_{\ell}\right\}} \frac{\left(\xi_{\ell}+1-t\right) !}{(z-t) !\left(\xi_{\ell}+1-z\right) !} \widetilde{y}_{\ell-\frac{3}{2}}^{\left(\xi_{\ell-1}-2 \xi_{\ell}+4 t\right)} \widetilde{y}_{2 \ell-4}^{\left(\xi_{\ell}+1-t\right)} \widetilde{y}_{2 \ell-2}^{\left(\xi_{\ell}-t\right)} v_{\boldsymbol{\xi}}=0 .
$$

Our aim is to show that each summand in $(4.20)$ vanishes. Let $v=\left(v_{0}, \ldots, v_{\xi_{\ell}}\right)$, where

$$
v_{t}=\widetilde{y}_{\ell-\frac{3}{2}}^{\left(\xi_{\ell-2}-\xi_{\ell}+4 t\right)} \widetilde{y}_{2 \ell-4}^{\left(\xi_{\ell}+1-t\right)} \widetilde{y}_{2 \ell-2}^{\left(\xi_{\ell}-t\right)} v_{\boldsymbol{\xi}}, \quad 0 \leq t \leq \xi_{\ell} .
$$

By using (4.20) for $z=1, \ldots, \xi_{\ell}+1$ we get a system of linear equations $A v=0$ where $A$ is invertible. Hence $v=0$. The proof of the remaining parts is similar and will be omitted. 


\section{Proof of Proposition 2.4}

In this section, we establish a recursive formulae for the graded multiplicities and prove Proposition 2.4.

5.1. The following lemma is the analogue of [6, Lemma 3.8] and is proven similarly using Corollary 4.7. The proof will be omitted.

Lemma. Let $\boldsymbol{\xi} \in \mathcal{P}_{\ell}, \ell>1$, and $\bar{\xi}$ be the unique partition obtained from $\boldsymbol{\xi}$ by removing $\xi_{0}$. For $s \in \mathbb{Z}_{+}$, we have

$$
\left[V(\boldsymbol{\xi}): D\left(\xi_{0}, s\right)\right]_{q}=q^{2(|\boldsymbol{\xi}|-s)}\left[V(\overline{\boldsymbol{\xi}}): D\left(\xi_{0}, s-\xi_{0}\right)\right]_{q}
$$

5.2. Now we are able to give a recursive formulae for the graded multiplicities.

Proposition. Let $m, n \in \mathbb{N}$.

(1) We write $s=m s_{1}+s_{0}$, where $0<s_{0} \leq m$ and set $\boldsymbol{\xi}=\left((m+1), m^{s_{1}}, s_{0}\right)$.

(i) If $2 s_{0} \leq m$ and $s_{1} \geq 1$, then

$$
\begin{aligned}
{[D(m, s): D(m+1, n)]_{q} } & =q^{2(s-n)}[D(m, s-m-1): D(m+1, n-m-1)]_{q} \\
& +q^{4 s_{1} s_{0}}\left[D\left(m, s-2 s_{0}\right): D(m+1, n)\right]_{q} .
\end{aligned}
$$

(ii) If $2 s_{0}>m$ and $s_{1} \geq 1$, then

$$
\begin{aligned}
{[D(m, s): D(} & m+1, n)]_{q}=q^{2(s-n)}[D(m, s-m-1): D(m+1, n-m-1)]_{q} \\
& +q^{\left(2 s_{1}+1\right)\left(2 s_{0}-m\right)}\left[D\left(m, s+m-2 s_{0}\right): D(m+1, n)\right]_{q} \\
& +\sum_{j=1}^{k(\boldsymbol{\xi})} q^{2 s_{1}\left(2 s_{0}-j\right)+j+m-2 n}\left[D\left(m, s-2 s_{0}+j-1\right): D(m+1, n-m-1)\right]_{q} .
\end{aligned}
$$

(2) For $j \in \mathbb{Z}_{+}, 0 \leq n, k \leq m$ and $m j+k \geq n$,

$$
[D(m, m j+k): D(m+1, n)]_{q}= \begin{cases}q^{j(m j+2 k)} & \text { if } n=k, \\ q^{(j+1)(m j+2 k-m)} & \text { if } n=m-k, \\ 0 & \text { otherwise. }\end{cases}
$$

Proof. Using the recursive formulae given in Corollary 4.7 and Lemma 5.1 we get in part (i)

$$
\begin{aligned}
{[D(m, s): D(m+1, n)]_{q} } & =q^{2(s-n)}[D(m, s-m-1): D(m+1, n-m-1)]_{q} \\
& +q^{4 s_{1} s_{0}}\left[D\left(m+\delta_{s_{1}, 1}, s-2 s_{0}\right): D(m+1, n)\right]_{q} \\
& +\delta_{n, m+1} \delta_{s_{0}, 1} \delta_{s_{1}, 2} q^{3 m}+\left(1-\delta_{2 s_{0}, m}\right) \delta_{s_{0}, n} \delta_{s_{1}, 1} q^{m+2 s_{0}}
\end{aligned}
$$

The statement follows from the following identities, which are easy consequences of (4.3):

$$
\left[D\left(m, s-2 s_{0}\right): D(m+1, n)\right]_{q}=\left[D\left(m+\delta_{s_{1}, 1}, s-2 s_{0}\right): D(m+1, n)\right]_{q}+\left(1-\delta_{2 s_{0}, m}\right) \delta_{s_{0}, n} \delta_{s_{1}, 1} q^{m-2 n}
$$

and

$$
\begin{aligned}
{[D(m, s-m-1): D(m+1, n-m-1)]_{q} } & =\left[D\left(m+\delta_{s_{1}, 2} \delta_{s_{0}, 1}, s-m-1\right): D(m+1, n-m-1)\right]_{q} \\
& +\delta_{n, m+1} \delta_{s_{0}, 1} \delta_{s_{1}, 2} q^{m} .
\end{aligned}
$$


The proof of part (ii) follows exactly the the same ideas and is left to the reader. Now we prove part (2) of the proposition. Note that the case $j=0$ follows from (4.3). So by induction we can assume that

$$
\left[D\left(m, m j^{\prime}+k\right): D(m+1, n)\right]_{q}= \begin{cases}q^{j^{\prime}\left(m j^{\prime}+2 k\right)} & \text { if } n=k, \\ q^{\left(j^{\prime}+1\right)\left(m j^{\prime}+2 k-m\right)} & \text { if } n=m-k, \\ 0 & \text { otherwise. }\end{cases}
$$

holds for all $0 \leq j^{\prime}<j, 0 \leq n, k \leq m$ such that $m j^{\prime}+k \geq n$. If $k=0$, we can assume that $j>1$, since $j=1$ follows once more with (4.3). Using the recursive formulae from part (1) and the induction hypothesis we get

$$
\begin{aligned}
{[D(m, m j): D(m+1, n)]_{q} } & =q^{m(2 j-1)}[D(m,(j-1) m): D(m+1, n)]_{q} \\
& = \begin{cases}q^{j(m j)} & \text { if } n=0, \\
q^{(j+1)(m j-m)} & \text { if } n=m, \\
0 & \text { otherwise. }\end{cases}
\end{aligned}
$$

If $0<k \leq \frac{m}{2}$, then

$$
\begin{aligned}
{[D(m, m j+k): D(m+1, n)]_{q} } & =q^{4 k j}[D(m, m j-k): D(m+1, n)]_{q} \\
& = \begin{cases}q^{j(m j+2 k)} & \text { if } n=k, \\
q^{(j+1)(m j+2 k-m)} & \text { if } n=m-k, \\
0 & \text { otherwise. }\end{cases}
\end{aligned}
$$

If $\frac{m}{2}<k \leq m$, then

$$
\begin{aligned}
{[D(m, m j+k): D(m+1, n)]_{q} } & =q^{(2 j+1)(2 k-m)}[D(m, m j+m-k): D(m+1, n)]_{q} \\
& =q^{(2 j+1)(2 k-m)} q^{4(m-k) j}[D(m, m(j-1)+k): D(m+1, n)]_{q} \\
& = \begin{cases}q^{j(m j+2 k)} & \text { if } n=k, \\
q^{(j+1)(m j+2 k-m)} & \text { if } n=m-k, \\
0 & \text { otherwise. }\end{cases}
\end{aligned}
$$

5.3. Proof of Proposition 2.4(i). If $s \in\{0,1\}$ the statement of the proposition follows from Proposition 5.2(2). Also the case $p=0$ is clear. Using the recursive formulae in Proposition 5.2, an easy induction argument shows

$$
\begin{aligned}
{[D(1, s+p): D(2, s)]_{q} } & \left.=q^{2 p}[D(1,(s-2)+p): D(2, s-2)]_{q}+q^{2(s+p)-1}[D(1, s+(p-1)): D(2, s))\right]_{q} \\
& =q^{p\left(s+p+\operatorname{res}_{2}(s)\right)}\left[\begin{array}{c}
\left\lfloor\frac{s-2}{2}\right\rfloor+p \\
p
\end{array}\right]_{q^{2}}+q^{2 s+p} q^{(p-1)\left(s+p+\operatorname{res}_{2}(s)\right)}\left[\begin{array}{c}
\left\lfloor\frac{s}{2}\right\rfloor+p-1 \\
p-1
\end{array}\right]_{q^{2}} \\
& =q^{p\left(s+p+\operatorname{res}_{2}(s)\right)}\left(\left[\begin{array}{c}
\left\lfloor\frac{s-2}{2}\right\rfloor+p \\
p
\end{array}\right]_{q^{2}}+q^{s-\operatorname{res}_{2}(s)}\left[\begin{array}{c}
\left\lfloor\frac{s}{2}\right\rfloor+p-1 \\
p-1
\end{array}\right]_{q^{2}}\right) \\
& =q^{p\left(s+p+\operatorname{res}_{2}(s)\right)}\left[\begin{array}{c}
\left\lfloor\frac{s}{2}\right\rfloor+p \\
p
\end{array}\right]_{q^{2}},
\end{aligned}
$$

which finishes the proof. 
5.4. Proof of Proposition 2.4(ii). Recall the following recursive formulae from Proposition 5.2:

$$
\begin{gathered}
{[D(2,2 n): D(3, s)]_{q}=q^{2(2 n-s)}[D(2,2 n-3): D(3, s-3)]_{q}+q^{2(2 n-1)}[D(2,2 n-2): D(3, s)]_{q}} \\
+q^{6 n-2 s-3}[D(2,2 n-4): D(3, s-3)]_{q}, \quad \text { if } n \geq 2, \\
{[D(2,2 n+1): D(3, s)]_{q}=q^{4 n+2-2 s}[D(2,2 n-2): D(3, s-3)]_{q}} \\
+q^{4 n}[D(2,2 n-1): D(3, s)]_{q}, \quad \text { if } n \geq 1 .
\end{gathered}
$$

Since all cases follow the same idea, we will only prove

$$
\begin{aligned}
& {[D(2,3 s+r+2 p): D(3,3 s+r)]_{q}=} \\
& \quad q^{2\left(p^{2}+p(2 s+r)\right)} \sum_{j=0}^{p} q^{2 j\left(j+\delta_{r, 1}\right)}\left[\begin{array}{c}
s+p-j \\
s
\end{array}\right]_{q^{2}}\left[\begin{array}{c}
\left\lfloor\frac{s}{2}\right\rfloor+j-\delta_{r, 1} \delta_{\operatorname{res}_{2}(s), 0} \\
2 j
\end{array}\right]_{q^{2}},
\end{aligned}
$$

where $r \in\{0,1,2\}$ and $s \in \mathbb{Z}_{+}$. The proof proceeds by induction on $s$. If $s=0$, the statement follows from Proposition 5.2(2) and the induction begins. The strategy of the proof is to show that the $q$-binomial formulae in (5.1) satisfies the above recursive formulae. Let us define

$$
u^{\prime}=\delta_{r, 1} \operatorname{res}_{2}(s), \quad y^{\prime}=\left(\delta_{r, 0}+\delta_{r, 2}\right) \delta_{\operatorname{res}_{2}(s), 0} .
$$

Case 1: In this case we suppose that $3 s+r$ is even. By the induction hypothesis, it will be enough to show that the $q$-binomial formula in (5.1) satisfies the recursive

$$
\begin{aligned}
{[D(2,3 s+r+2 p): D(3,3 s+r)]_{q} } & =q^{4 p}[D(2,3(s-1)+r+2 p): D(3,3(s-1)+r)]_{q} \\
& +q^{2(3 s+r+2 p-1)}[D(2,3 s+r+2(p-1)): D(3,3 s+r)]_{q} \\
& +q^{3 s+r+6 p-3}[D(2,3(s-1)+r+2(p-1)+1): D(3,3(s-1)+r)]_{q} .
\end{aligned}
$$

Equivalently, it suffices to show that the following sum vanishes for all $p \geq 0$ :

$$
\begin{aligned}
& \sum_{j=0}^{p} q^{2 j\left(j+\delta_{r, 1}\right)}\left[\begin{array}{c}
s+p-j-1 \\
s-1
\end{array}\right]_{q^{2}}\left[\begin{array}{c}
\left\lfloor\frac{s-1}{2}\right\rfloor+j-u^{\prime} \\
2 j
\end{array}\right]_{q^{2}} \\
&+\sum_{j=0}^{p} q^{2 s+2 j\left(j+\delta_{r, 1}\right)}\left[\begin{array}{c}
s+p-j-1 \\
s
\end{array}\right]_{q^{2}}\left[\begin{array}{c}
\left\lfloor\frac{s}{2}\right\rfloor+j \\
2 j
\end{array}\right]_{q^{2}} \\
&+\sum_{j=0}^{p-1} q^{2 j\left(j+\delta_{r, 1}\right)+2 j+s+\delta_{r, 1}}\left[\begin{array}{c}
s+p-j-2 \\
s-1
\end{array}\right]_{q^{2}}\left[\begin{array}{c}
\left\lfloor\frac{s-1}{2}\right\rfloor+j+y^{\prime} \\
2 j+1
\end{array}\right]_{q^{2}} \\
&-\sum_{j=0}^{p} q^{2 j\left(j+\delta_{r, 1}\right)}\left[\begin{array}{c}
s+p-j \\
s
\end{array}\right]_{q^{2}}\left[\begin{array}{c}
\left\lfloor\frac{s}{2}\right\rfloor+j \\
2 j
\end{array}\right]_{q^{2}} .
\end{aligned}
$$

Note that $y^{\prime}=1$ implies $u^{\prime}=0$ and $s$ is even and $y^{\prime}=0$ forces $u^{\prime}=1$ and $s$ is odd. It follows

$$
\left[\begin{array}{c}
\left\lfloor\frac{s-1}{2}\right\rfloor+j-u^{\prime} \\
2 j
\end{array}\right]_{q^{2}}+q^{s-2 j-\delta_{r, 1}}\left[\begin{array}{c}
\left\lfloor\frac{s-1}{2}\right\rfloor+j+y^{\prime}-1 \\
2 j-1
\end{array}\right]_{q^{2}}=\left[\begin{array}{c}
\left\lfloor\frac{s}{2}\right\rfloor+j \\
2 j
\end{array}\right]_{q^{2}}, \quad 1 \leq j \leq p .
$$

Now rewriting the third term in the above sum as

$$
\sum_{j=1}^{p} q^{2 j\left(j+\delta_{r, 1}\right)+s-2 j-\delta_{r, 1}}\left[\begin{array}{c}
s+p-j-1 \\
s-1
\end{array}\right]_{q^{2}}\left[\begin{array}{c}
\left\lfloor\frac{s-1}{2}\right\rfloor+j+y^{\prime}-1 \\
2 j-1
\end{array}\right]_{q^{2}}
$$


we obtain the desired result.

Case 2: We assume that $3 s+r$ is odd. Again, it will be enough to show that the $q$-binomial formula in (5.1) satisfies the recursive

$$
\begin{aligned}
{[D(2,3 s+r+2 p): D(3,3 s+r)]_{q} } & =q^{4 p}[D(2,3(s-1)+r+2 p): D(3,3(s-1)+r)]_{q} \\
& +q^{6 s+2 r+4 p-2}[D(2,3 s+r+2 p-2): D(3,3 s+r)]_{q},
\end{aligned}
$$

which is equivalent to the statement that the following sum vanishes for all $p \geq 0$ :

$$
\begin{aligned}
& \sum_{j=0}^{p} q^{2 j\left(j+\delta_{r, 1}\right)}\left(\left[\begin{array}{c}
s+p-j-1 \\
s-1
\end{array}\right]_{q^{2}}\left[\begin{array}{c}
\left\lfloor\frac{s-1}{2}\right\rfloor+j-u^{\prime} \\
2 j
\end{array}\right]_{q^{2}}+q^{2 s}\left[\begin{array}{c}
s+p-j-1 \\
s
\end{array}\right]_{q^{2}}\left[\begin{array}{c}
\left\lfloor\frac{s}{2}\right\rfloor+j-\delta_{r, 1} \delta_{\operatorname{res}_{2}(s), 0} \\
2 j
\end{array}\right]_{q^{2}}\right. \\
& \left.-\left[\begin{array}{c}
s+p-j \\
s
\end{array}\right]_{q^{2}}\left[\begin{array}{c}
\left\lfloor\frac{s}{2}\right\rfloor+j-\delta_{r, 1} \delta_{\operatorname{res}_{2}(s), 0} \\
2 j
\end{array}\right]_{q^{2}}\right) .
\end{aligned}
$$

Since $\left\lfloor\frac{s-1}{2}\right\rfloor-u^{\prime}=\left\lfloor\frac{s}{2}\right\rfloor-\delta_{r, 1} \delta_{\operatorname{res}_{2}(s), 0}$ it is easy to see that the above sum is zero. Hence Proposition 2.4(ii) is proven.

\section{Proof of Theorem 2}

In this section, we study the generating series $A_{n}^{m^{\prime} \rightarrow m}(x)$ for the numerical multiplicities and give a closed formula when $m^{\prime}=1$ and $m=m^{\prime}+1$, showing that they are rational functions. We will use freely the notation and results established in the previous sections.

6.1. The following result gives a recursive formulae. It will be convinient to set $A_{-1}^{1 \rightarrow m}(x)=1$.

Proposition. For $m \geq 2$ and $n \geq-1$ write $n+1=m n_{1}+n_{0}$, where $0<n_{0} \leq m, n_{1} \geq-1$. The generating series $A_{n}^{1 \rightarrow m}(x)$ satisfies the reccurence,

$$
A_{n}^{1 \rightarrow m}(x)= \begin{cases}A_{n+1}^{1 \rightarrow m}(x) & \text { if } 2 n_{0}=m-1, \\ A_{n+1}^{1 \rightarrow m}(x)\left(1-\left(1-\delta_{m, 2}\right) x\right) & \text { if } n_{0}=m-1 \text { or } 2 n_{0}=m-2, \\ A_{n+1}^{1 \rightarrow m}(x)-\left(1-\delta_{m, 2}\right) x^{2} A_{n+2}^{1 \rightarrow m}(x) & \text { if } 2 n_{0}=m, \\ (1-x) A_{n+1}^{1 \rightarrow m}(x)-\left(1-\delta_{m, 2}\right) x^{2} A_{n+2}^{1 \rightarrow m}(x) & \text { if } 2 n_{0} \notin\{m-2, m-1, m\} \text { and } n_{0} \neq m-1 .\end{cases}
$$

The proof of the above proposition is postponed to the end of this section. We first discuss how this can be used to prove Theorem 2 .

6.2. Proof of Theorem 2. Set $D_{m}(x)=a_{m}(x) a_{m+1}(x)$ and $F_{k}=A_{k}^{1 \rightarrow m}(x)$ for $k \geq-1$. The theorem follows if we prove that for all $k \geq 0$ and $0 \leq r<m$, we have

$$
(a) F_{m k+r}=N_{m, r}(x) F_{m k+m-1}, \quad(b) F_{m k+m-1}=\frac{1}{D_{m}(x)^{\left\lfloor\frac{k}{m}\right\rfloor+1}},
$$

where

$$
N_{m, r}(x)= \begin{cases}a_{2 m-2 r-1}(x) & \text { if }\left\lfloor\frac{m}{2}\right\rfloor \leq r \leq m-1, \\ a_{m}(x) a_{m-2 r-1}(x) & \text { if } 0 \leq r \leq\left\lfloor\frac{m}{2}\right\rfloor-1 .\end{cases}
$$

We first prove (a). If $r=m-1$ this is immediate since $N_{m, m-1}(x)=1$ and if $r=m-2$ this follows from the second case of Proposition 6.1. Assume now that we have proved the equality for all $0 \leq r^{\prime}<m$ with $r^{\prime}>r$ and $r<m-2$. To prove the equality for $r$, we use Proposition 6.1 and the following equalities which can be easily checked

- $N_{m,\left\lfloor\frac{m}{2}\right\rfloor-1}(x)=N_{m,\left\lfloor\frac{m}{2}\right\rfloor}(x)$, if $\mathrm{m}$ is odd,

- $N_{m, m-2}(x)=\left(1-\left(1-\delta_{m, 2}\right) x\right) N_{m, m-1}(x)$,

- $N_{m, \frac{m}{2}-2}(x)=(1-x) N_{m, \frac{m}{2}-1}(x)$ if $m \geq 4$ is even, 
- $N_{m, \frac{m}{2}-1}(x)=N_{m, \frac{m}{2}}(x)-\left(1-\delta_{m, 2}\right) x^{2} N_{m, \frac{m}{2}+1}(x)$ if $\mathrm{m}$ is even,

- $N_{m, p-1}(x)=(1-x) N_{m, p}(x)-x^{2} N_{m, p+1}(x)$ if $2 p \notin\{m-2, m-1, m\}$ and $1 \leq p \leq m-2$,

- $N_{m, m-1}(x) D_{m}(x)=(1-x) N_{m, 0}(x)-\left(1-\delta_{m, 2}\right) x^{2} N_{m, 1}(x)$.

Now part (a) follows by an easy induction argument. In order to prove (b), observe that the last case of Proposition 6.1 gives

$$
F_{m(k-1)+m-1}=(1-x) F_{m(k-1)+m}-\left(1-\delta_{m, 2} x^{2}\right) x^{2} F_{m(k-1)+m+1}=D_{m}(x) F_{m k+m-1}, k \geq 0 .
$$

Since $F_{-1}=1$ we get $D_{m}(x)^{k+1} F_{m k+m-1}=1$ and the proof is complete.

6.3. The following lemma is needed in the proof of Proposition 6.1.

Lemma. Let $m \in \mathbb{N}$ and $p \in \mathbb{Z}_{+}$. Write $p=m p_{1}+p_{0}$ with $p_{1}, p_{0} \in \mathbb{Z}, p_{1} \geq-1$ and $0<p_{0} \leq m$. Then

$$
\begin{aligned}
\operatorname{ch}_{\mathfrak{g}} D(m, p) \operatorname{ch}_{\mathfrak{g}} D(1,1)=\operatorname{ch}_{\mathfrak{g}} D(m, p+1) & +\left(1-\delta_{m, 1}\right)\left(\left(1-\delta_{2 p_{0}, m}-\delta_{2 p_{0}, m+1}-\delta_{p_{0}, m}\right) \operatorname{ch}_{\mathfrak{g}} D(m, p-1)\right. \\
& \left.+\left(1-\delta_{2 p_{0}, m}-\delta_{2 p_{0}, m-1}\right) \operatorname{ch}_{\mathfrak{g}} D(m, p)\right) .
\end{aligned}
$$

Proof. If $m=1$, the statement is obvious since $D(1, p) \otimes D(1,1) \cong_{\mathfrak{g}} D(1, p+1)$; also if $p=0$ there is nothing to prove. So assume from now on $m \geq 2$ and $p \neq 0$.

Case 1: Let $p_{0}<m$. Then $D(m, p) \cong_{\mathfrak{g}} D\left(m, m p_{1}\right) \otimes D\left(m, p_{0}\right)$ and hence

$$
\begin{aligned}
D(m, p) \otimes D(1,1) & \cong_{\mathfrak{g}} D\left(m, m p_{1}\right) \otimes D\left(m, p_{0}\right) \otimes D(1,1) \\
& \simeq_{\mathfrak{g}} D\left(m, m p_{1}\right) \otimes D\left(m, p_{0}\right) \otimes(D(m, 1) \oplus V(0)) \\
& \cong_{\mathfrak{g}} D\left(m, m p_{1}\right) \otimes\left(D\left(m, p_{0}\right) \otimes D(m, 1) \oplus D\left(m, p_{0}\right)\right) .
\end{aligned}
$$

So the lemma in this case follows if we prove

$$
\begin{aligned}
\operatorname{ch}_{\mathfrak{g}} D\left(m, p_{0}\right) & \operatorname{ch}_{\mathfrak{g}} D(m, 1)=\operatorname{ch}_{\mathfrak{g}} D\left(m, p_{0}+1\right) \\
& +\left(1-\delta_{2 p_{0}, m}-\delta_{2 p_{0}, m+1}\right) \operatorname{ch}_{\mathfrak{g}} D\left(m, p_{0}-1\right)-\left(\delta_{2 p_{0}, m}+\delta_{2 p_{0}, m-1}\right) \operatorname{ch}_{\mathfrak{g}} D\left(m, p_{0}\right) .
\end{aligned}
$$

We have the following decomposition into irreducible $\mathfrak{g}$-modules:

$$
\begin{aligned}
D\left(m, p_{0}\right) \otimes D(m, 1) & \cong \begin{cases}\left(V\left(p_{0}\right) \oplus V\left(p_{0}-1\right) \oplus \cdots \oplus V\left(m-p_{0}\right)\right) \otimes V(1), & \text { if } 2 p_{0}>m, \\
V\left(p_{0}\right) \otimes V(1), & \text { if } 2 p_{0} \leq m,\end{cases} \\
& \cong \begin{cases}\bigoplus_{j=m-p_{0}+1}^{p_{0}+1} V(j) \oplus \bigoplus_{j=m-p_{0}-1}^{p_{0}-1} V(j), & \text { if } 2 p_{0}>m, \\
V\left(p_{0}+1\right) \oplus V\left(p_{0}-1\right), & \text { if } 2 p_{0} \leq m .\end{cases}
\end{aligned}
$$

Further we know

$$
\begin{aligned}
& D\left(m, p_{0}+1\right) \cong \begin{cases}V\left(p_{0}+1\right) \oplus V\left(p_{0}\right) \oplus \cdots \oplus V\left(m-p_{0}-1\right), & \text { if } 2 p_{0}+2>m \\
V\left(p_{0}+1\right), & \text { if } 2 p_{0}+2 \leq m .\end{cases} \\
& D\left(m, p_{0}-1\right) \cong \begin{cases}V\left(p_{0}-1\right) \oplus V\left(p_{0}\right) \oplus \cdots \oplus V\left(m-p_{0}+1\right), & \text { if } 2 p_{0}+2>m \\
V\left(p_{0}-1\right), & \text { if } 2 p_{0}+2 \leq m .\end{cases}
\end{aligned}
$$

Now writing both sides of (6.1) in terms of characters of irreducible $\mathfrak{g}$-modules and using the above formulae we get (6.1).

Case 2: Let $p_{0}=m$. We get

$$
D(m, p) \otimes D(1,1) \cong_{\mathfrak{g}} D\left(m, m\left(p_{1}+1\right)\right) \otimes(D(m, 1) \oplus V(0)) \cong_{\mathfrak{g}} D(m, p+1) \oplus D(m, p) .
$$


6.4. Proof of Proposition 6.1. We continue assuming $p=m p_{1}+p_{0}$ with $p_{1}, p_{0} \in \mathbb{Z}, p_{1} \geq-1$ and $0<p_{0} \leq m$. For $s \in \mathbb{Z}_{+}$, we write

$$
\operatorname{ch}_{\mathfrak{g}} D(1, s)=\sum_{p \geq 0}[D(1, s): D(m, p)]_{q=1} \operatorname{ch}_{\mathfrak{g}} D(m, p)
$$

and multiply both sides with $\operatorname{ch}_{\mathfrak{g}} D(1,1)$. We get

$$
\operatorname{ch}_{\mathfrak{g}} D(1, s) \operatorname{ch}_{\mathfrak{g}} D(1,1)=\operatorname{ch}_{\mathfrak{g}} D(1, s+1)=\sum_{p \geq 0}[D(1, s): D(m, p)]_{q=1} \operatorname{ch}_{\mathfrak{g}} D(m, p) \operatorname{ch}_{\mathfrak{g}} D(1,1)
$$

Now we can apply Lemma 6.3 to both sides of (6.2). Applying to the right hand side gives a linear combination of characters of level $m$ Demazure modules. Writing the left side as

$$
\operatorname{ch}_{\mathfrak{g}} D(1, s+1)=\sum_{p \geq 0}[D(1, s+1): D(m, p)]_{q=1} \operatorname{ch}_{\mathfrak{g}} D(m, p)
$$

and equating the coefficients on both sides of (6.2) gives

$$
\begin{aligned}
{[D(1, s+1): D(m, p)]_{q=1} } & =[D(1, s): D(m, p-1)]_{q=1}+ \\
& +\left(1-\delta_{2 \widetilde{p_{0}}, m}-\delta_{2 \widetilde{p_{0}} \cdot m+1}-\delta_{\widetilde{p_{0}}, m}\right)[D(1, s): D(m, p+1)]_{q=1} \\
& +\left(1-\delta_{2 p_{0}, m}-\delta_{2 p_{0}, m-1}\right)[D(1, s): D(m, p)]_{q=1}
\end{aligned}
$$

where

$$
\widetilde{p_{0}}= \begin{cases}p_{0}+1, & \text { if } p_{0}<m, \\ 1, & \text { otherwise }\end{cases}
$$

Let us consider the case $2 p_{0}=m$. We get from above

$$
[D(1, s+1): D(m, p)]_{q=1}=[D(1, s): D(m, p-1)]_{q=1}+\left(1-\delta_{m, 2}\right)[D(1, s): D(m, p+1)]_{q=1}
$$

and thus

$$
\begin{aligned}
A_{p-1}^{1 \rightarrow m}(x) & =1+\sum_{k>0}[D(1, p-1+k): D(m, p-1)] x^{k} \\
& =1+\sum_{k>0}[D(1, p+k): D(m, p)] x^{k}-\left(1-\delta_{m, 2}\right) \sum_{k>0}[D(1, p-1+k): D(m, p+1)] x^{k} \\
& =A_{p}^{1 \rightarrow m}(x)-\left(1-\delta_{m, 2}\right) x^{2} A_{p+1}^{1 \rightarrow m}(x) .
\end{aligned}
$$

Repeating the same argument for all remaining cases gives the statement of Proposition 6.1. We omit the details.

6.5. Finally, we turn our attention to the study of $A_{n}^{m \rightarrow m+1}(x)$ for $m \geq 1$. 
Proposition. For $m \geq 1$ and $n \geq 0$, write $n=(m+1) p_{n}-r_{n}$ where $p_{n} \in \mathbb{Z}_{+}$and $0 \leq r_{n} \leq m$. Then,

$$
A_{n}^{m \rightarrow m+1}(x)= \begin{cases}A_{n+m}^{m \rightarrow m+1}(x)-x^{2 r_{n}} A_{n+2 r_{n}}^{m \rightarrow m+1}(x) & \text { if } 1 \leq r_{n} \leq\left\lfloor\frac{m-1}{2}\right\rfloor, \\ A_{n+m}^{m \rightarrow m+1}(x)-x^{2 r_{n}-m} A_{n+2 r_{n}}^{m \rightarrow m+1}(x) & \text { if } r_{n}=\left\lfloor\frac{m+1}{2}\right\rfloor \text { and } m \text { is odd, } \\ A_{n+m}^{m \rightarrow m+1}(x)-x^{2 r_{n}} A_{n+2 r_{n}}^{m \rightarrow m+1}(x) & \text { if } r_{n}=\left\lfloor\frac{m+1}{2}\right\rfloor \text { and } m \text { is even, } \\ A_{n \rightarrow m}^{m \rightarrow m+1}(x)-x^{2 r_{n}-m} A_{n+2 r_{n}}^{m \rightarrow m+1}(x)-x^{2 r_{n}-m-1} A_{n+2 r_{n}-m-1}(x) & \text { if }\left\lfloor\frac{m+3}{2}\right\rfloor \leq r_{n} \leq m, \\ A_{n+m}^{m \rightarrow m+1}(x) & \text { if } m+1 \mid n .\end{cases}
$$

Proof. To simplify notation, we fix $m \geq 1$ and for $s, n \in \mathbb{Z}_{+}$, set

$$
\nu(s, n)=[D(m, s): D(m+1, n)]_{q=1} .
$$

Recall that $\nu(s, n)=0$ if $s<n$. The theorem follows if we prove for all $s, n \geq 0$ that

$$
\nu(s, n)= \begin{cases}\nu(s+m, n+m)-\nu\left(s, n+2 r_{n}\right) & \text { if } 1 \leq r_{n} \leq\left\lfloor\frac{m-1}{2}\right\rfloor, \\ \nu(s+m, n+m)-\nu\left(s+m, n+2 r_{n}\right) & \text { if } r_{n}=\left\lfloor\frac{m+1}{2}\right\rfloor \text { and } m \text { is odd, } \\ \nu(s+m, n+m)-\nu\left(s, n+2 r_{n}\right) & \text { if } r_{n}=\left\lfloor\frac{m+1}{2}\right\rfloor \text { and } m \text { is even, } \\ \nu(s+m, n+m)-\nu\left(s+m, n+2 r_{n}\right)-\nu\left(s, n+2 r_{n}-m-1\right) & \text { if }\left\lfloor\frac{m+3}{2}\right\rfloor \leq r_{n} \leq m, \\ \nu(s+m, n+m) & \text { if } m+1 \mid n .\end{cases}
$$

Notice that this equality holds whenever $s<n$, since both sides are zero. Hence we can assume that $s \geq n$. Taking $q=1$ in Proposition 5.2 gives

$$
s \geq 0,0 \leq n \leq m \Longrightarrow \nu(s, n)= \begin{cases}1 & \text { if } s+n \text { or } s-n \text { is a multiple of } m, \\ 0 & \text { otherwise }\end{cases}
$$

Applying once more Proposition 5.2 with $s=m s_{1}+s_{0}, 0<s_{0} \leq m, s_{1} \geq 1$ and $\boldsymbol{\xi}=\left((m+1), m^{s_{1}}, s_{0}\right)$ gives

$$
\begin{aligned}
\nu(s, n)=\nu(s-m-1, n-m-1) & +\nu\left(s+m-2 s_{0}, n\right) \\
& +\sum_{j=1}^{k(\boldsymbol{\xi})} \nu\left(s-2 s_{0}+j-1, n-m-1\right), \text { if } 2 s_{0}>m
\end{aligned}
$$

and

$$
\nu(s, n)=\nu(s-m-1, n-m-1)+\nu\left(s-2 s_{0}, n\right), \quad \text { if } 2 s_{0} \leq m .
$$

We now proceed to prove equation (6.4) by induction on $n$. Equation (6.5) obviously implies $\nu(s, 0)=$ $\nu(s+m, m)$ and hence induction begins. Now let $n>0$. Assume that $\nu\left(s, n^{\prime}\right)$ satisfies (6.4) for all $0 \leq n^{\prime}<n$ and for all $s \in \mathbb{Z}_{+}$. We proceed by induction on $s$ to prove that $\nu(s, n)$ satisfies (6.4) for all $s \in \mathbb{Z}_{+}$. Notice that the $s=0$ is clear since both sides of (6.4) are zero. Further, as remarked earlier, we assume for the rest of the proof that $s \geq n$.

Case 1: Suppose $0<n \leq m$ and $1 \leq r_{n} \leq\left\lfloor\frac{m-1}{2}\right\rfloor$. We have to prove that

$$
\nu(s, n)=\nu(s+m, n+m)-\nu(s, 2 m+2-n) .
$$


Case 1(a): Suppose $s \geq m+1$ and $2 s_{0}>m$. Then the recursive can be used for both terms of the right hand side and we get

$$
\begin{gathered}
\nu(s+m, n+m)=\nu(s-1, n-1)+\nu\left(s+2 m-2 s_{0}, n+m\right)+\sum_{j=1}^{k(\boldsymbol{\xi})} \nu\left(s+m-2 s_{0}+j-1, n-1\right), \\
\begin{aligned}
\nu(s, 2 m+2-n)=\nu(s-m-1, m+1-n)+\nu\left(s+m-2 s_{0}, 2 m+2-n\right) \\
+\sum_{j=1}^{k(\boldsymbol{\xi})} \nu\left(s-2 s_{0}+j-1, m+1-n\right) .
\end{aligned}
\end{gathered}
$$

Set

$$
\begin{gathered}
T_{1}=\nu(s-1, n-1)-\nu(s-m-1, m+1-n), \\
T_{2}=\nu\left(s+2 m-2 s_{0}, n+m\right)-\nu\left(s+m-2 s_{0}, 2 m+2-n\right),
\end{gathered}
$$

and

$$
T_{3}=\nu\left(s+m-2 s_{0}+j-1, n-1\right)-\nu\left(s-2 s_{0}+j-1, m+1-n\right) .
$$

Equation (6.5) applies to both terms in $T_{1}$ and $T_{3}$. Since

$$
\begin{aligned}
(s-1)-(n-1) & =(s-m-1)+(m+1-n) \\
(s-1)+(n-1) & \equiv(s-m-1)-(m+1-n) \quad(\bmod m), \\
\left(s+m-2 s_{0}+j-1\right)-(n-1) & =\left(s-2 s_{0}+j-1\right)+(m+1-n) \\
\left(s+m-2 s_{0}+j-1\right)+(n-1) & \equiv\left(s-2 s_{0}+j-1\right)-(m+1-n) \quad(\bmod m),
\end{aligned}
$$

we deduce that $T_{1}=T_{3}=0$. Further, since $s-2 s_{0}<s$, the inductive hypothesis gives $T_{2}=$ $\nu\left(s+m-2 s_{0}, n\right)$. Hence we have to verify $\nu(s, n)=\nu\left(s+m-2 s_{0}, n\right)$. Since $s \equiv s_{0}(\bmod m)$, we obtain $s+m-2 s_{0} \equiv-s(\bmod m)$ and thus $s \pm n \equiv-\left(s+m-2 s_{0} \mp n\right)(\bmod m)$. Applying $(6.5)$ completes the proof.

Case 1(b):Suppose that $s \geq m+1$ and $2 s_{0} \leq m$. Then the recursive formulae can be used for both terms on the right hand side and we get

$$
\begin{gathered}
\nu(s+m, n+m)=\nu(s-1, n-1)+\nu\left(s-2 s_{0}+m, n+m\right), \\
\nu(s, 2 m+2-n)=\nu(s-m-1, m+1-n)+\nu\left(s-2 s_{0}, 2 m+2-n\right) .
\end{gathered}
$$

Since $T_{1}=0$ and $s-2 s_{0}<s$, by using induction on $s$, we get

$$
\nu\left(s-2 s_{0}+m, n+m\right)-\nu\left(s-2 s_{0}, 2 m+2-n\right)=\nu(s, n)
$$

which proves the claim.

Case 1(c): Suppose $s \leq m$. Since $2 m+2-n>m$, we have $\nu(s, 2 m+2-n)=0$. Thus we need to show that $\nu(s, n)=\nu(s+m, n+m)$. Applying the recursive formulae again we get

$$
\nu(s+m, n+m)=\nu(s-1, n-1)+\nu\left(s+2 m-2 s_{0}, n+m\right) .
$$

But since $0<s \leq m$, we have $s=s_{0}$, and hence $s+2 m-2 s_{0}=2 m-s_{0}<n+m$. Thus the second term vanishes. It remains to show $\nu(s-1, n-1)=\nu(s, n)$. But this is clear by (6.5), which implies for $1 \leq s, n \leq m: \nu(s-1, n-1)=\nu(s, n)=\delta_{s, n}$.

Case 2: Suppose $n \geq m+1$ and $1 \leq r_{n} \leq\left\lfloor\frac{m-1}{2}\right\rfloor$. Then $m+1 \nmid r$. Consider

$$
S=\nu(s+m, n+m)-\nu\left(s, n+2 r_{n}\right)-\nu(s, n) .
$$


Case2(a): Assume that $2 s_{0}>m$. By applying the recursive to each of the terms of $S$, we have

$$
\begin{aligned}
S & =\nu(s-1, n-1)+\nu\left(s+2 m-2 s_{0}, n+m\right)+\sum_{j=1}^{k(\boldsymbol{\xi})} \nu\left(s+m-2 s_{0}+j-1, n-1\right) \\
& -\nu\left(s-m-1, n+2 r_{n}-m-1\right)-\nu\left(s+m-2 s_{0}, n+2 r_{n}\right)-\sum_{j=1}^{k(\boldsymbol{\xi})} \nu\left(s-2 s_{0}+j-1, n+2 r_{n}-m-1\right) \\
& -\nu(s-m-1, n-m-1)-\nu\left(s+m-2 s_{0}, n\right)-\sum_{j=1}^{k(\boldsymbol{\xi})} \nu\left(s-2 s_{0}+j-1, n-m-1\right) .
\end{aligned}
$$

Since $n-m-1<n, s+m-2 s_{0}<s$ and $s-2 s_{0}+j-1<s$, the inductive hypothesis gives

$$
\begin{aligned}
\nu(s-m-1, n-m-1) & =\nu(s-1, n-1)-\nu\left(s-m-1, n+2 r_{n}-m-1\right), \\
\nu\left(s+m-2 s_{0}, n\right) & =\nu\left(s+2 m-2 s_{0}, n+m\right)-\nu\left(s+m-2 s_{0}, n+2 r_{n}\right), \\
\nu\left(s-2 s_{0}+j-1, n-m-1\right) & =\nu\left(s+m-2 s_{0}+j-1, n-1\right)-\nu\left(s-2 s_{0}+j-1, n+2 r_{n}-m-1\right) .
\end{aligned}
$$

Substituting these equations in our equation for $S$, we obtain $S=0$.

Case2(b): Let us assume that $2 s_{0} \leq m$. By applying the recursive to each of the terms of $S$,

$$
\begin{array}{r}
S=\nu(s-1, n-1)+\nu\left(s+m-2 s_{0}, n+m\right)-\nu\left(s-m-1, n+2 r_{n}-m-1\right) \\
-\nu\left(s-2 s_{0}, n+2 r_{n}\right)-\nu(s-m-1, n-m-1)-\nu\left(s-2 s_{0}, n\right) .
\end{array}
$$

Again by using the induction hypothesis we get

$$
\begin{array}{r}
\nu(s-m-1, n-m-1)=\nu(s-1, n-1)-\nu\left(s-m-1, n+2 r_{n}-m-1\right), \\
\nu\left(s-2 s_{0}, n\right)=\nu\left(s+m-2 s_{0}, n+m\right)-\nu\left(s-2 s_{0}, n+2 r_{n}\right) .
\end{array}
$$

Substituting these equations in our equation for $S$, we obtain $S=0$. The remaining cases can be proven similarly and we leave the details to the reader.

6.6. We shall use Proposition 6.5 to establish a closed formula for the generating series $A_{n}^{m \rightarrow m+1}(x)$. For this we define polynomials $d_{n}(x), n \geq 0$ with non-negative integer coefficients as follows. For $0 \leq n \leq m$ set

$$
d_{n}(x)= \begin{cases}1 & \text { if } n=0, m, \\ 1+x^{m-2 n} & \text { if } 1<n \leq\left\lceil\frac{m-2}{2}\right\rceil, \\ 1+\delta_{r e s_{2}(m), 1} x & \text { if } n=\left\lceil\frac{m-1}{2}\right\rceil, \\ 1+x^{2 m-2 n} & \text { if }\left\lceil\frac{m}{2}\right\rceil \leq n \leq m-1 .\end{cases}
$$

The polynomials $d_{n}(x)$ for $n>m$ are defined by requiring that the following equality holds for all $p \geq 1$ :

$$
\left[\begin{array}{lllll}
d_{(m+1) p}(x) & d_{(m+1) p+1}(x) & \cdots & d_{(m+1) p+m}(x)
\end{array}\right]^{T}=K^{p}\left[\begin{array}{llll}
d_{0}(x) & d_{1}(x) & \cdots & d_{m}(x)
\end{array}\right]^{T},
$$

where $K$ is a $(m+1) \times(m+1)$ matrix defined as:

$$
K= \begin{cases}K_{1}+K_{2,0} & \text { if } m \text { is even } \\ K_{1}+K_{2,1} & \text { if } m \text { is odd }\end{cases}
$$


and the three $(m+1) \times(m+1)$ matrices $K_{1}, K_{2,0}$ and $K_{2,1}$ are defined below. Set

$$
K_{1}=\left[\begin{array}{cccccccc}
0 & 1 & & & & & & \\
& 0 & 1 & & & & & \\
& & \ddots & \ddots & & & & \\
& & & 0 & 1 & & & \\
& & & x^{m-1} & 1 & & \\
& & & & \ddots & \ddots & \\
& & & & & x^{m-1} & 1 \\
& & & & & & x^{m-1}
\end{array}\right]
$$

where number of zeros on the diagonal of the matrix $K_{1}$ is $\left\lceil\frac{m+1}{2}\right\rceil$ and the number of entries equal to $x^{m-1}$ is $\left\lfloor\frac{m+1}{2}\right\rfloor$. Set

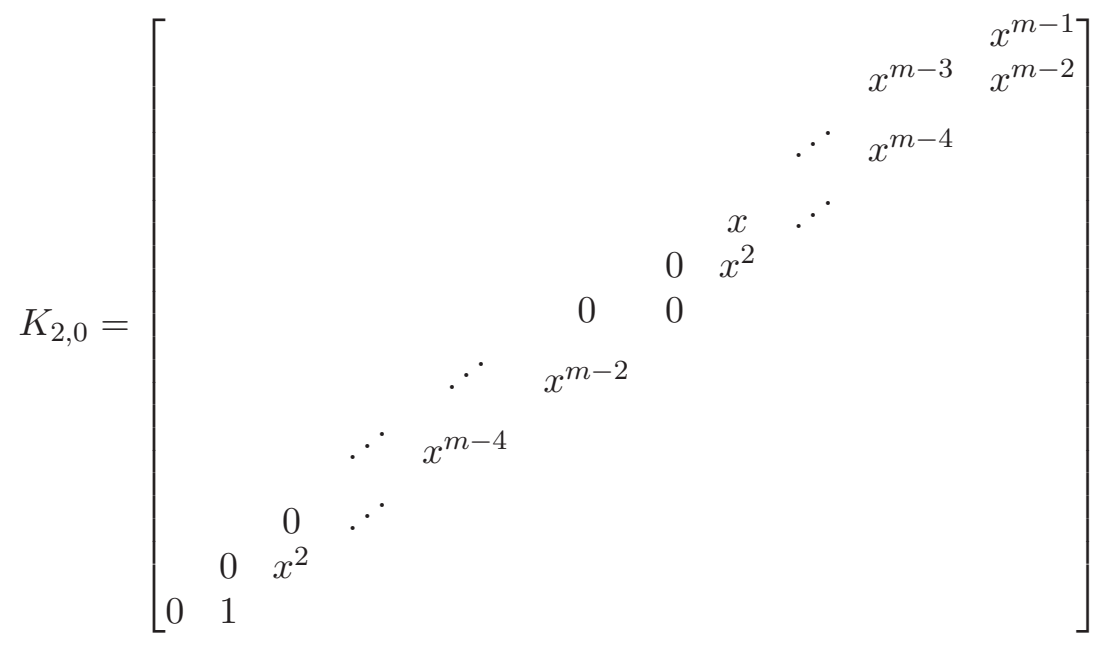

where the number of antigonal elements equal to zero in the matrix $K_{2,0}$ is equal to $\left\lceil\frac{m+1}{2}\right\rceil$. Set

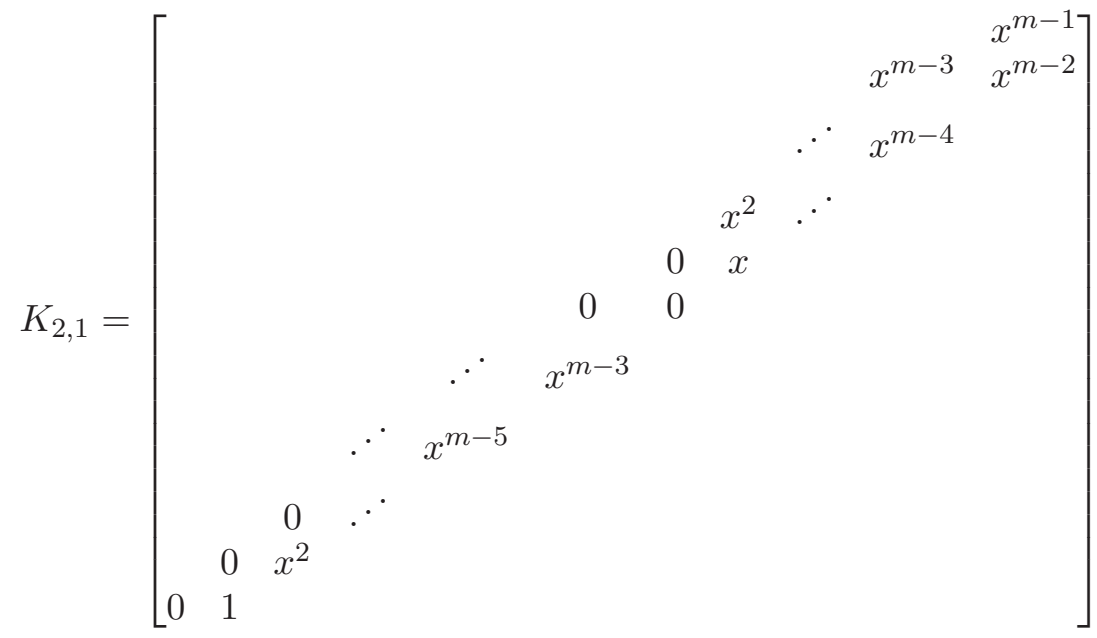

where the number of antigonal elements equal to zero in the matrix $K_{2,1}$ is equal to $\left\lceil\frac{m+3}{2}\right\rceil$.

Theorem 4. Let $m \geq 1$. Then, for all $n \geq 0$, we have

$$
A_{n}^{m \rightarrow m+1}(x)=\frac{d_{n}(x)}{\left(1-x^{m}\right)^{\left\lfloor\frac{n}{m+1}\right\rfloor+1}} .
$$


Proof. Let $n, p_{n} \in \mathbb{Z}_{+}, 0 \leq r_{n} \leq m$ such that $n=(m+1) p_{n}-r_{n}$. To simplify the notation in the proof we set $A_{n}:=A_{n}^{m \rightarrow m+1}(x)$ and

$$
e_{n}(x)=A_{n}\left(1-x^{m}\right)^{\left\lfloor\frac{n}{m+1}\right\rfloor+1} .
$$

The strategy of the proof is to show by induction on $p$ that

$$
\left[\begin{array}{lllll}
e_{(m+1) p}(x) & e_{(m+1) p+1}(x) & \cdots & e_{(m+1) p+m}(x)
\end{array}\right]^{T}=K^{p}\left[\begin{array}{llll}
d_{0}(x) & d_{1}(x) & \cdots & d_{m}(x)
\end{array}\right]^{T} .
$$

From Proposition 5.2, it is easy to see that

$$
A_{n}=\frac{d_{n}(x)}{\left(1-x^{m}\right)^{\left\lfloor\frac{n}{m+1}\right\rfloor+1}} \quad \text { for } 0 \leq n \leq m
$$

and the induction begins. First we verify certain recursive formulae for the $e_{n}(x)$ 's using Theorem 6.5. Case 1: If $1 \leq r_{n} \leq\left\lfloor\frac{m-1}{2}\right\rfloor$, we have

$$
\begin{gathered}
A_{(m+1) p_{n}-r_{n}}=A_{(m+1) p_{n}+m-r_{n}}-x^{2 r_{n}} A_{(m+1) p_{n}+r_{n}}, \\
A_{(m+1) p_{n}-\left(m-r_{n}\right)}=A_{(m+1) p_{n}+r_{n}}-x^{m-2 r_{n}} A_{(m+1) p_{n}+\left(m-r_{n}\right)}-x^{m-2 r_{n}-1} A_{(m+1) p_{n}-r_{n}-1} .
\end{gathered}
$$

Multiplying equation (6.8) by $x^{m-2 r_{n}}$ and adding the result to equation (6.9) gives

$$
\left(1-x^{m}\right) A_{(m+1) p_{n}+r_{n}}=A_{(m+1) p_{n}-\left(m-r_{n}\right)}+x^{m-2 r_{n}} A_{(m+1) p_{n}-r_{n}}+x^{m-2 r_{n}-1} A_{(m+1) p_{n}-r_{n}-1} .
$$

It follows

$$
e_{(m+1) p_{n}+r_{n}}(x)=e_{(m+1) p_{n}-\left(m-r_{n}\right)}(x)+x^{m-2 r_{n}} e_{(m+1) p_{n}-r_{n}}(x)+x^{m-2 r_{n}-1} e_{(m+1) p_{n}-r_{n}-1}(x) .
$$

Case 2: If $\left\lfloor\frac{m+3}{2}\right\rfloor \leq r_{n} \leq m-1$ we obtain

$$
\begin{aligned}
A_{(m+1) p_{n}-r_{n}}= & A_{(m+1) p_{n}+m-r_{n}}-x^{2 r_{n}-m} A_{(m+1) p_{n}+r_{n}}-x^{2 r_{n}-m-1} A_{(m+1) p_{n}+r_{n}-m-1}, \\
& A_{(m+1) p_{n}-\left(m-r_{n}\right)}=A_{(m+1) p_{n}+r_{n}}-x^{2 m-2 r_{n}} A_{(m+1) p_{n}+m-r_{n}} .
\end{aligned}
$$

Multiplying equation (6.11) by $x^{2 m-2 r_{n}}$ and adding the result to equation (6.12) gives

$$
\left(1-x^{m}\right) A_{(m+1) p_{n}+r_{n}}=A_{(m+1) p_{n}-\left(m-r_{n}\right)}+x^{2 m-2 r_{n}} A_{(m+1) p_{n}-r_{n}}^{m \rightarrow m+1}(x)+x^{m-1} A_{(m+1) p_{n}+r_{n}-m-1} .
$$

This implies

$$
e_{(m+1) p_{n}+r_{n}}(x)=e_{(m+1) p_{n}-\left(m-r_{n}\right)}(x)+x^{2 m-2 r_{n}} e_{(m+1) p_{n}-r_{n}}(x)+x^{m-1} e_{(m+1)\left(p_{n}-1\right)+r_{n}}(x) .
$$

Case 3: If $r_{n}=m$, then

$$
\begin{aligned}
A_{(m+1) p_{n}-m} & =A_{(m+1) p_{n}}-x^{m} A_{(m+1) p_{n}+m}-x^{m-1} A_{(m+1) p_{n}-1} \\
& =\left(1-x^{m}\right) A_{(m+1) p_{n}+m}-x^{m-1} A_{(m+1) p_{n}-1} .
\end{aligned}
$$

Hence

$$
e_{(m+1) p_{n}+m}(x)=e_{(m+1)\left(p_{n}-1\right)+1}(x)+x^{m-1} e_{(m+1)\left(p_{n}-1\right)+m}(x) .
$$

Case 4: If $m$ is even and $r_{n}=\frac{m}{2}$, we get

This shows

$$
\begin{aligned}
A_{(m+1) p_{n}-\frac{m}{2}} & =A_{(m+1) p_{n}+\frac{m}{2}}-x^{m} A_{(m+1) p_{n}+\frac{m}{2}} \\
& =\left(1-x^{m}\right) A_{(m+1) p_{n}+\frac{m}{2}} .
\end{aligned}
$$

$$
e_{(m+1) p_{n}+\frac{m}{2}}(x)=e_{(m+1)\left(p_{n}-1\right)+\frac{m}{2}+1}(x) .
$$

Case 4: If $m$ is odd, then we get the following two equations by setting $r_{n}=\left\lfloor\frac{m+1}{2}\right\rfloor$ and $r_{n}=\frac{m-1}{2}$ respectively:

$$
A_{(m+1) p_{n}-\frac{m+1}{2}}=A_{(m+1) p_{n}+\frac{m-1}{2}}-x A_{(m+1) p_{n}+\frac{m+1}{2}}^{m \rightarrow m+1}(x)
$$




$$
A_{(m+1) p_{n}-\frac{m-1}{2}}=A_{(m+1) p_{n}+\frac{m+1}{2}}-x^{m-1} A_{(m+1) p_{n}+\frac{m-1}{2}} .
$$

Multiplying equation (6.17) by $x$ and adding the result to equation (6.16) yields

$$
\left(1-x^{m}\right) A_{(m+1) p_{n}+\frac{m-1}{2}}=A_{(m+1) p_{n}-\frac{m+1}{2}}^{m \rightarrow m+1}(x)+x A_{(m+1) p_{n}-\frac{m-1}{2}} .
$$

Thus

$$
e_{(m+1) p_{n}+\frac{m-1}{2}}(x)=e_{(m+1) p_{n}-\frac{m+1}{2}}(x)+x e_{(m+1) p_{n}-\frac{m-1}{2}}(x) .
$$

Similarly multiplying equation (6.16) by $x^{m}$ and adding the result to equation (6.17) gives

$$
\left(1-x^{m}\right) A_{(m+1) p_{n}+\frac{m+1}{2}}=A_{(m+1) p_{n}-\frac{m-1}{2}}^{m \rightarrow m+1}(x)+x^{m-1} A_{(m+1) p_{n}-\frac{m+1}{2}},
$$

which implies

$$
e_{(m+1) p_{n}+\frac{m+1}{2}}(x)=e_{(m+1)\left(p_{n}-1\right)+\frac{m+1}{2}+1}(x)+x^{m-1} e_{(m+1)\left(p_{n}-1\right)+\frac{m+1}{2}}(x) .
$$

Hence the recursive formulae for the $e_{n}(x)$ 's from equations $(6.10),(6.13),(6.14),(6.15),(6.18)$ and (6.19) imply for $p>0$

$$
\begin{array}{r}
K\left[\begin{array}{llll}
e_{(m+1)(p-1)}(x) & e_{(m+1)(p-1)+1}(x) & \cdots & e_{(m+1)(p-1)+m}(x)
\end{array}\right]^{T} \\
=\left[\begin{array}{llll}
e_{(m+1) p}(x) & e_{(m+1) p+1}(x) & \cdots & e_{(m+1) p+m}(x)
\end{array}\right]^{T} .
\end{array}
$$

Applying the induction hypothesis shows (6.7).

\section{REFERENCES}

[1] George E. Andrews. Fibonacci numbers and the Rogers-Ramanujan identities. Fibonacci Quart., 42(1):3-19, 2004.

[2] Rekha Biswal, Vyjayanthi Chari, Lisa Schneider, and Sankaran Viswanath. Demazure flags, Chebyshev polynomials, partial and mock theta functions. J. Combin. Theory Ser. A, 140:38-75, 2016.

[3] Rekha Biswal, Vyjayanthi Chari, Perri Shereen, and Jeffrey Wand. Cone theta functions and Demazure flags in higher rank. in preparation.

[4] L. Carlitz. Fibonacci notes. IV. q-Fibonacci polynomials. Fibonacci Quart., 13:97-102, 1975.

[5] Vyjayanthi Chari, Bogdan Ion, and Deniz Kus. Weyl modules for the hyperspecial current algebra. Int. Math. Res. Not. IMRN, (15):6470-6515, 2015.

[6] Vyjayanthi Chari, Lisa Schneider, Perri Shereen, and Jeffrey Wand. Modules with Demazure Flags and Character Formulae. SIGMA Symmetry Integrability Geom. Methods Appl., 10, 2014.

[7] Vyjayanthi Chari and R. Venkatesh. Demazure modules, fusion products and Q-systems. Comm. Math. Phys., 333(2):799-830, 2015.

[8] Michel Demazure. Une nouvelle formule des caractères. Bull. Sci. Math. (2), 98(3):163-172, 1974.

[9] Boris Feigin and Sergei Loktev. On generalized Kostka polynomials and the quantum Verlinde rule. In Differential topology, infinite-dimensional Lie algebras, and applications, volume 194 of Amer. Math. Soc. Transl. Ser. 2, pages 61-79. Amer. Math. Soc., Providence, RI, 1999.

[10] Bogdan Ion. Nonsymmetric Macdonald polynomials and Demazure characters. Duke Math. J., 116(2):299-318, 2003.

[11] Anthony Joseph. Modules with a Demazure flag. In Studies in Lie theory, volume 243 of Progr. Math., pages 131-169. Birkhäuser Boston, Boston, MA, 2006.

[12] Victor G. Kac. Infinite-dimensional Lie algebras. Cambridge University Press, Cambridge, third edition, 1990.

[13] Shrawan Kumar. Kac-Moody groups, their flag varieties and representation theory, volume 204 of Progress in Mathematics. Birkhäuser Boston Inc., Boston, MA, 2002.

[14] Deniz Kus and R. Venkatesh. Twisted Demazure modules, fusion product decomposition and twisted $Q$-systems. Represent. Theory, 20:94-127, 2016.

[15] J. Lepowsky and R. L. Wilson. The structure of standard modules, I: Universal algebras and the Rogers-Ramanujan identities,. Invent. Math., 77:199-290, 1984.

[16] Peter Littelmann. Crystal graphs and Young tableaux. J. Algebra, 175(1):65-87, 1995.

[17] Olivier Mathieu. Construction du groupe de Kac-Moody et applications. C. R. Acad. Sci. Paris Sér. I Math., 306(5):227-230, 1988. 
[18] Katsuyuki Naoi. Weyl modules, Demazure modules and finite crystals for non-simply laced type. Adv. Math., 229(2):875-934, 2012.

[19] Yasmine B. Sanderson. On the connection between Macdonald polynomials and Demazure characters. J. Algebraic Combin., 11(3):269-275, 2000.

[20] Issai Schur. Gesammelte Abhandlungen. Band II. Springer-Verlag, Berlin-New York, 1973. Herausgegeben von Alfred Brauer und Hans Rohrbach.

[21] Lucy Joan Slater. Generalized hypergeometric functions. Cambridge University Press, Cambridge, 1966.

The Institute of Mathematical Sciences, Chennai, India

E-mail address: rekha@imsc.res.in

Department of Mathematics, University of California, Riverside, CA 92521

E-mail address: chari@math.ucr.edu

Mathematisches Institut, Universität Bonn, Germany

E-mail address: dkus@math.uni-bonn.de 